Journal for ImmunoTherapy of Cancer

\title{
Helicobacter pylori infection disturbs the tumor immune microenvironment and is associated with a discrepant prognosis in gastric de novo diffuse large B-cell lymphoma
}

\author{
Yuwei Deng, ${ }^{1}$ Wenjia Su, ${ }^{2}$ Junwen Zhu, ${ }^{1}$ Hongfei $\mathrm{Ji}^{3}$ Xiaoping Zhou, ${ }^{1}$ \\ Jingshu Geng, ${ }^{4}$ Jiayu Zhu, ${ }^{1}$ Qingyuan Zhang (D) ${ }^{1}$
}

To cite: Deng Y, Su W, Zhu J, et al. Helicobacter pylori infection disturbs the tumor immune microenvironment and is associated with a discrepant prognosis in gastric de novo diffuse large B-cell lymphoma. Journal for ImmunoTherapy of Cancer 2021;9:e002947. doi:10.1136/jitc-2021-002947

- Additional supplemental material is published online only. To view, please visit the journal online (http://dx.doi.org/10. 1136/jitc-2021-002947).

YD and WS contributed equally. Accepted 09 September 2021

Check for updates

(c) Author(s) (or their employer(s)) 2021. Re-use permitted under CC BY-NC. No commercial re-use. See rights and permissions. Published by BMJ.

For numbered affiliations see end of article.

Correspondence to Dr Qingyuan Zhang; 0566@hrbmu.edu.cn

\section{ABSTRACT}

Background Gastric diffuse large B-cell lymphoma (gDLBCL) related to Helicobacter pylori infection exhibits a wide spectrum of prognosis, and the tumor immune microenvironment (TIME) affects tumor progression. However, there are few studies on the correlation between prognosis and changes of TIME induced by $H$. pylori infection in de novo gDLBCL.

Methods A retrospective study was performed to determine the prognostic value of TIME related to $H$. pylori infection in de novo gDLBCL. A total of 252 patients were included and have been treated with standard rituximab to cyclophosphamide, doxorubicin, vincristine, and prednisone chemotherapy or other similar regimens in addition to $H$. pylori eradication (HPE). All patients were stratified by $H$. pylori infection, HPE efficacy, and preliminary TIME evaluation using conventional criteria. Statistical analyses were conducted. To assess the mechanism, 30 subjects were assessed for $H$. pylori infection. The components and spatial distributions of TIME were analyzed.

Results The median follow-up of the 252 patients was 66.6 months (range 0.7-119.2), and the 5-year overall survival (OS) was $78.0 \%$. A total of $109 \mathrm{H}$. pyloripositive cases with pathological complete remission and high tumor-infiltrating T lymphocytes (cohort 1) had significantly higher 5 -year progression-free survival (88.1\% vs $70.5 \%, \mathrm{p}<0.001)$ and 0 S $(89.2 \%$ vs $76.6 \%$, $\mathrm{p}<0.001$ ) than the other 143 patients (cohort 2). Among 30 patients, 19 were cytotoxin-associated gene Amarked as the cohort 1 subset. Compared with cohort 2 , cohort 1 exhibited increased inflammatory factors (tumor necrosis factor- $\alpha$, interferon $\gamma$, etc) and decreased immunosuppressive components (PD-L1, PD-1, IL-10, etc). There was reduced NF-kB activation. Cancerpromoting immune cells (PD-1hiTim-3+ CTL, Tregs, M2-like macrophages, etc) occupied a minor spatial distribution, while the antitumor subtypes increased, corresponding to favorable survival.

Conclusion $H$. pylori-evoked inflammatory responses disturb the TIME, causing a differential prognosis in de novo gDLBCL, which can be used to identify patients who could benefit from HPE and immunochemotherapy.

\section{INTRODUCTION}

Diffuse large B cell lymphoma (DLBCL), which is the leading type of aggressive nonHodgkin's lymphoma from activated B-cell (ABC) and germinal center B-cell (GCB) origins, arises from extranodal organs in about $30 \%-45 \%$ of cases. The stomach is the most common site, whereby DLBCL accounts for $<5 \%$ of all gastric malignancies. ${ }^{12}$ Primary gastric DLBCL (de novo gDLBCL) is a heterogeneous disease lacking mucosaassociated lymphoid tissue (MALT) characteristics, which constitutes approximately $30 \%$ of all gastric lymphomas. ${ }^{3}$ In China, the overall prevalence of Helicobacter pylori infection in the gastrointestinal tract is $54 \%$, which is much higher than in other countries in which the figure stands at a maximum of $31 \%$. Several studies have established that $H$. pylori affects biological processes and the prognosis of gastric MALT lymphoma. $H$. pylori eradication (HPE) applied as the standard first-line treatment of MALT, results in $65 \%$ pathological complete remission (pCR), with a median of 7.7 years. ${ }^{5}$ Compared with Westerners, Asians exhibit a more favorable response rate $(84 \%$ vs $74 \%){ }^{6}$

At present, HPE combined with rituximab plus anthracycline-based immunochemotherapy (R-cyclophosphamide, doxorubicin, vincristine, and prednisone, CHOP) has reached clinical consensus due to its marked pCR and favorable overall survival (OS) in gDLBCL, with $50 \%$ pCR in earlystage patients. ${ }^{7}$ However, the initial failure, defined as local relapse, was found to be the most common pattern with systemic therapy (R-CHOP) alone, accounting for $44 \%-63 \%$ of all relapse cases in Asians. ${ }^{8}$ Although several studies have confirmed the 
correlation between $H$. pylori infection and prognosis in de novo gDLBCL, ${ }^{9}$ a worrying fluctuation of $27 \%-87 \%$ pCR has also been observed among the $H$. pylori-positive subgroup adopting HPE alone in the Chinese population, ${ }^{10}$ causing a delay of available immunotherapy due to a lack of pre-evaluation.

Latest studies have emphasized the reliable prognostic stratification based on immune cytotypes related to the tumor immune microenvironment (TIME), demonstrating that 'Stromal-1' and 'Stromal-2', respectively, predicted favorable and inferior outcomes in DLBCL patients with R-CHOP, regardless of the cell-of-origin. ${ }^{11}$ Numerous studies have suggested that tumor programmed death ligand-1 (PD-L1) expansion and nuclear factor kappa beta (NF-KB) growth factor signaling modulates programmed death protein-1 (PD-1)/PD-L1 immunosuppression and tumor progression, leading to an inferior prognosis in DLBCL. ${ }^{9} 12$ A parallel study has shown that H. pylori-positive de novo gDLBCL presenting pCR after HPE indicates a favorable prognosis, especially in the limited-stage subgroup. ${ }^{13} \mathrm{H}$. pylori infiltration can trigger effective inflammatory immune responses involving the recruitment and activation of dendritic cells, $\mathrm{CD} 4^{+} \mathrm{T}$ cells (Th1, Th17, and Tregs), neutrophils, and macrophages. ${ }^{14}$ However, there are few reports on the effect of TIME on the prognosis.

Here, we investigated the intriguing prognostic correlation by evaluating $H$. pylori infection with pCR after HPE treatment and the corresponding initial TIME status in de novo gDLBCL patients (especially early stage) in a major cohort. The results highlight that $H$. pylori-induced inflammatory disturbances in the TIME mapping to a wide distribution of phenotypically diverse immune cells may accurately reflect the prognostic differences in $H$. pylori-related de novo gDLBCL, which was defined by $H$. pylori-inherent cytotoxin-associated gene A (CagA) toxic factor. $^{15}$

\section{METHODS \\ Patients}

A total of 252 paraffin-embedded cancer specimens collected between 2005 and 2019 were retrieved from the Harbin Medical University Cancer Hospital and the First Affiliated Hospital of Harbin Medical University. All participants provided signed informed consent. Endoscopic biopsy and surgical resection were performed for the acquisition of pathological specimens, which were re-examined by experienced histopathologists to confirm the diagnosis according to the WHO hematological malignant tumor classification system. De novo gDLBCL was defined as excluding histological features of MALT lymphoma. Tumors of MALT-origin proof and secondary gastric lymphoma were excluded. Six cycles of standardized CHOP (cyclophosphamide, doxorubicin, vincristine, and prednisone) or CHOP-like chemotherapy accompanied by rituximab $(\mathrm{R})$ were administered to all the patients. Second-line chemotherapy or other treatments were also available based on indications. The total baseline clinical features of the patients according to medical records are shown in table 1.

\section{Helicobacter pylori infection}

At least one of the following tests was used to confirm the clinical presence of $H$. pylori infection: histological reports (Warthin-Starry staining was conducted in all cases), bacterial culture, and serological examination. HPE treatment was administered to all $H$. pylori-positive patients, comprizing bismuth compounds, proton pump inhibitors (PPI), and two selective antibiotics out of clarithromycin, amoxicillin, and metronidazole.

\section{Preliminary TIME assessment}

The stored slides, pathologically diagnosed using immunohistochemistry were retrieved to analyze tumorinfiltrating $\mathrm{T}$ lymphocytes (TIL-Ts), including all 76 surgical specimens and 176 biopsies. CD3 (routine clinicopathological marker) was used to define TIL-Ts. All CD $3^{+}$TIL-Ts counts were evaluated by two experienced pathologists, and were divided into high-TIL-Ts and lowTIL-Ts groups based on the cut-off value defined as $14 \%$, which was based on Receiver Operating Characteristic (ROC) curves according to a previous study. ${ }^{16}$

\section{Clinical parameters}

All parameters were retrieved from individual medical records. Physical examinations included peripheral lymph nodes, Waldeyer ring, specific hematological indexes, CT, gastroendoscopy, ultrasonography, positron emission tomography-CT, and bone marrow biopsy. For patients undergoing surgery, further consideration was given to intraoperative exploration and pathological authentication. The routine clinical pathological staining results for CD10, BCL6 and MUM-1 were used to classify all tumors into GCB or non-GCB subgroups by pathologists as described previously. ${ }^{9}$ The Lugano staging system was employed for staging and classification of the tumors. In addition, the performance status was evaluated according to the Eastern Cooperative Oncology Group (ECOG) criteria and International Prognostic Index (IPI). The response of each patient to HPE or chemotherapy was assessed using periodical endoscopic biopsy and other imaging examinations. Tumors that exhibited pCR were classified toward therapeutic effectiveness clinically.

\section{Immunohistochemical staining}

Thirty paraffin-embedded tissue slides were subjected to immunohistochemical staining using the following antibodies: PD-L1 (AG27557, Proteintech), PD-1 (AG12470, Proteintech), p65 (AG1199, Proteintech), TRAF6 (AG24296, Proteintech), CagA (A-10, Santa Cruz Biotechnology), tumor necrosis factor- $\alpha$ (TNF- $\alpha$ ) (A11534, ABclonal), interferon (IFN)- $\gamma$ (AG8321, Proteintech), and IL-10 (AG14870, Proteintech). Staining of preprocessed endoscopic biopsy samples and surgical specimens was carried out on embedded slices using the indirect peroxidase method ( $\mathrm{pH} 6.0$ in sodium citrate buffer). Two expert 


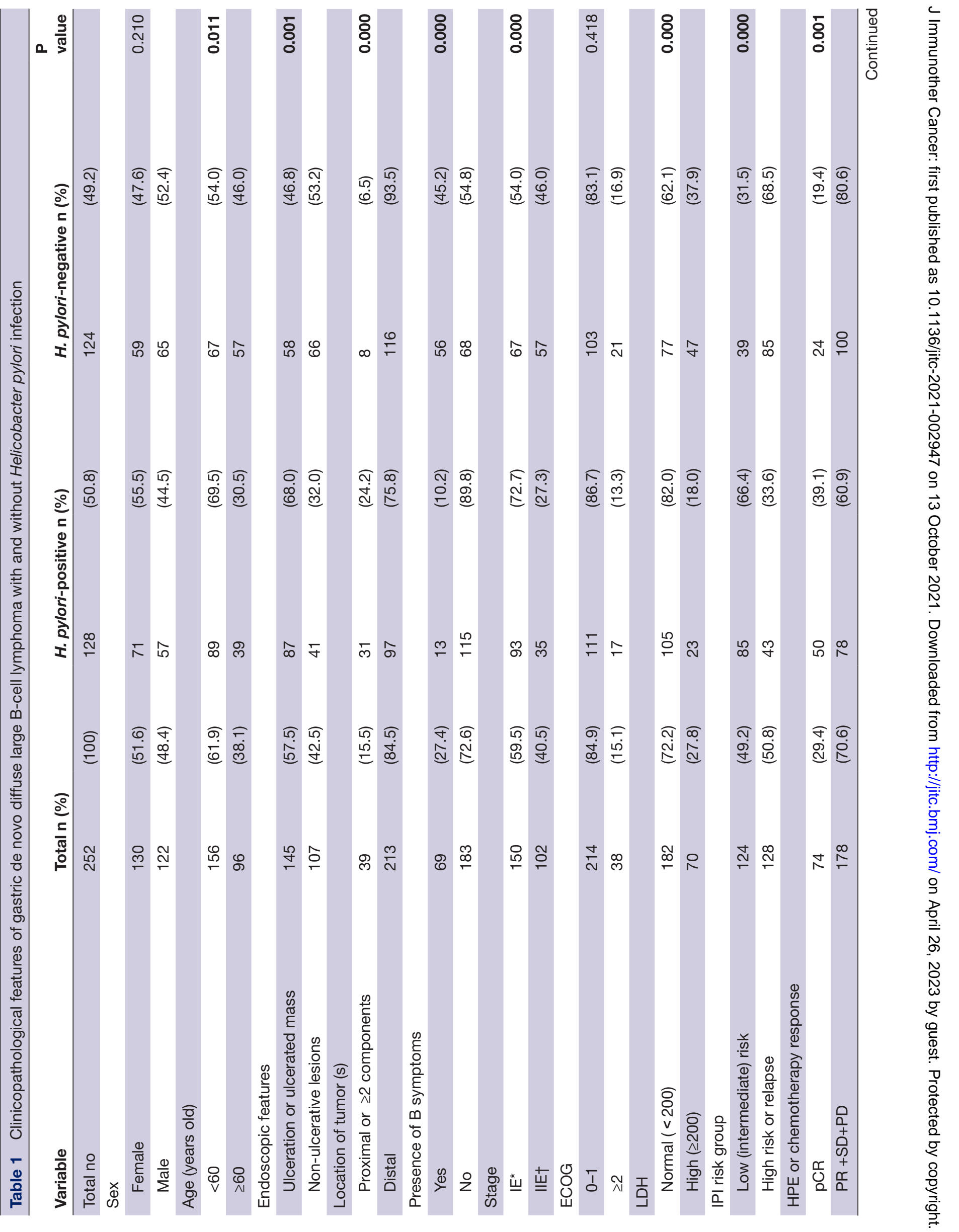




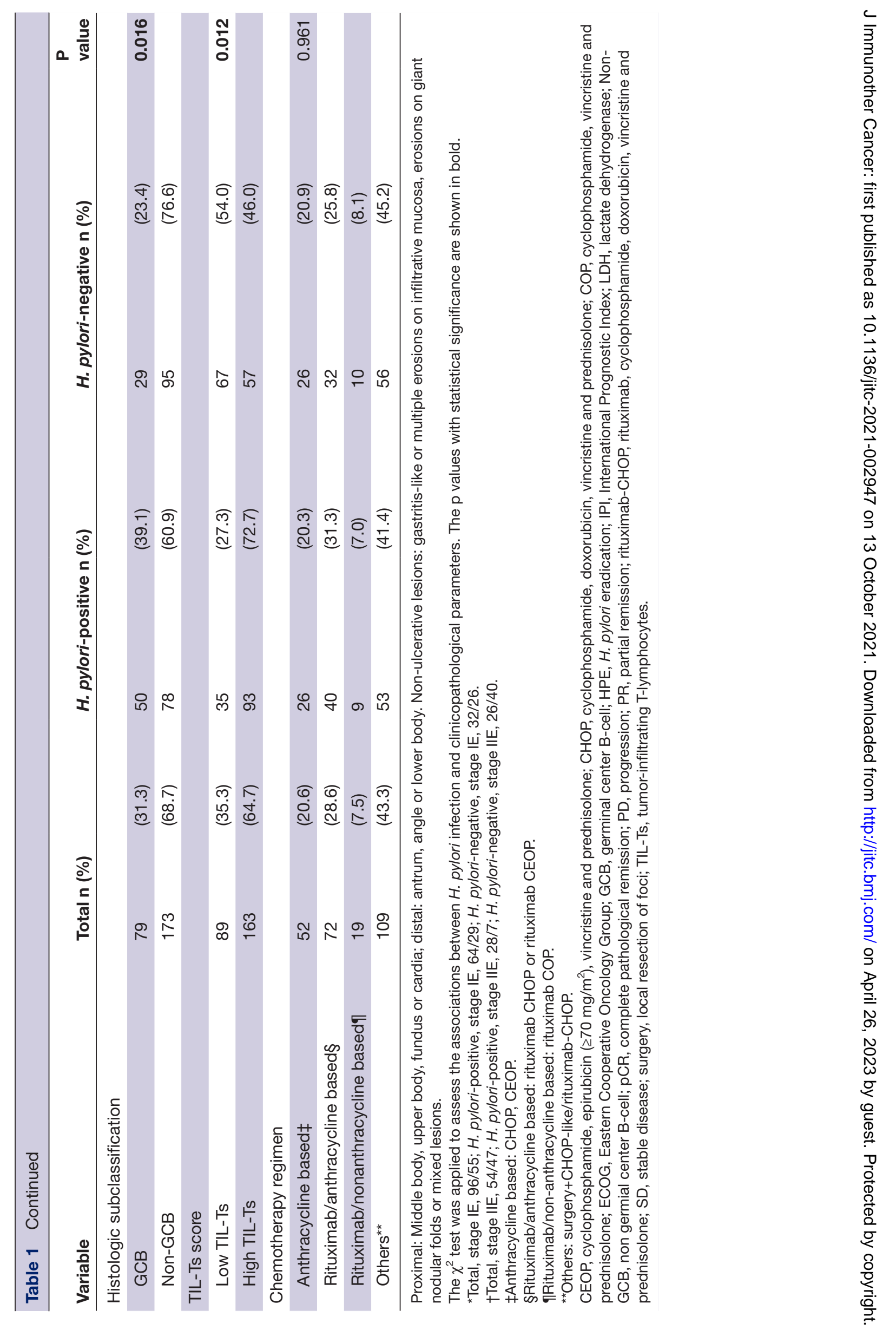


pathologists scored the slides independently, bypassing the clinical data. An IScope microscope (IS.1159EPLi, Euromex) and Image Focus V.4.0 (Euromex) software were applied to scan and capture the images. The H-score to quantify the P65, TRAF6, TNF- $\alpha$, IFN- $\gamma$, IL-10, PD-1, and PD-L1 staining intensities was calculated as follows: $\sum$ (3xpercentage of strong staining $+2 \times$ percentage of moderate staining +percentage of weak staining) using Image J with 0-300 fluctuations. The graphic data were displayed as mean $\pm \mathrm{SE}$ of the mean (SEM). For CagA markers, the positive expression exceeded $10 \%$ in cells with moderate or strong immunostaining. ${ }^{15}$

\section{Multiplexed ion beam imaging by time of flight (MIBI-TOF) tissue image \\ Antibodies and panels}

Lymphocyte types as well as activation, cytokines, vascular, and spatial structure of other tissue cells was based on all antibody panels. Staining with the primary antibodies (online supplemental table 1) with metal-conjugation was conducted using the Maxpar labeling kit (Fluidigm) using concentrations based on BioTek (Berten Instruments) at $0.5 \mathrm{~g} / \mathrm{L}$ and stored long-term at $4^{\circ} \mathrm{C}$.

\section{Tissue antibody labeling}

Formalin-fixed and paraffin-embedded tissue slides were stained as listed in online supplemental table 1. After dewaxing, antigen retrieval ( $\mathrm{R} \& \mathrm{D}$ systems) and blocking were performed. The samples were incubated with the antibody cocktail overnight at $4^{\circ} \mathrm{C}$ and then stained with Intercalator-Ir (Fluidigm, 201 192A) for the detection of DNA.

\section{Imaging mass cytometry}

According to H\&E staining, appropriate $500 \times 500 \mu \mathrm{m}$ sites were selected for scanning. IMC Images were captured using a multiplexed ion beam imaging by time of flight Imaging System (Fluidigm) at $200 \mathrm{~Hz}$ with interspersed capture of isotope polymers (Fluidigm) to monitor the stability. MCDViewer, CellProfiler, and HistoCAT were used for the processing of successful image captures.

\section{Single-cell identification}

The original MCD file saved in TIFF format was transformed using imctools (https://github.com/BodenmillerGroup/imctools). The data were segmented into single cells using CellProfiler V.3.1.8.

\section{Data transformation and normalization}

The single-cell markers were measured using histoCat v1.75, multiplied by a factor of $10^{7}$ to yield values larger than 1 and then log-transformed. The single-cell data were deleted at the 95 th percentile to exclude the outliers. The heatmaps were presented with $z$-scored means of clusters. Then, the data were standardized using Harmony in t-Distributed Stochastic Neighbor Embedding (t-SNE) and PhenoGraph.

\section{Clustering}

The aggregation of subpopulation groups according to their markers was performed using PhenoGraph (V.2.0) with 15 nearest neighbors clustering. Twenty defined clusters were assigned into a larger aggregation as the hierarchical clustering (Euclidean distance and Ward's linkage) according to their mean pertinence of related markers. The uncertainty of each subtree was assessed by multiscale bootstrap resampling ( $\mathrm{R}$ package pvclust, V.2.0).

\section{Barnes-Hut t-SNE}

Single-cell data with a high dimension was degraded into two dimensions by the nonlinear dimensionality reduction algorithm (t-SNE). The Barnes-Hut of t-SNE was harmoniously standardized to exclude the default parameters (perplexity=25).

\section{Neighborhood analysis}

The pairwise neighbor interactions for cell type significance involved in enrichment or depletion were detected using the CellProfiler Measure Object Neighbors module and neighborhood (https://github.com/BodenmillerGroup/neighbouRhood) functions accompanied by permutation-test-based analysis of the spatial distribution. Four pixels $(4 \mu \mathrm{m})$ showed the boundary of neighboring cells. Differences with $\mathrm{p}<0.01$ were identified as significant.

\section{Tandem mass tag peptide labeling proteomics}

Tandem mass tag peptide (TMT) Mass Tagging Kits and Reagents (Thermo Fisher Scientific) were applied for TMT labeling of the peptides with lysed (Sigma) samples. The tissues were fractionated and shotgun proteomics analyses were performed using an EASY-nLCTM 1200 ultrahigh-pressure liquid chromatography system (Thermo Fisher) interfaced with a $\mathrm{Q}$ Exactive HF (X) mass spectrometer (Thermo Fisher) operated in the datadependent acquisition (DDA) mode. The final spectra of each run were searched solely according to the homo_ sapiens_uniprot_2020_7_2.fasta (192 320 sequences) database by using the search software Proteome Discoverer V.2.2 (Thermo), followed by filtering of the retrieved data. The proteins that exhibited significantly different abundance between two groups were classified using the t-test, and the differentially expressed proteins were identified according to fold change (FC) $\geq 1.2$ and $\mathrm{p} \leq 0.05$ for the upregulated proteins and FC $\leq 0.83$ and $\mathrm{p} \leq 0.05$ for the downregulated proteins. Gene Ontology (GO), Clusters of Orthologous Groups, Kyoto Encyclopedia of Genes and Genomes (KEGG) and InterPro (IPR) functional analyses were carried out with the non-redundant protein databases (Pfam, PRINTS, ProDom, SMART, ProSite, PANTHER). Volcano plots and cluster heatmaps as well as GO, IPR, and KEGG enrichments were marked based on DPEs. The STRING-db server (http://string. embl.de/) was used to predict the probable proteinprotein interactions. 

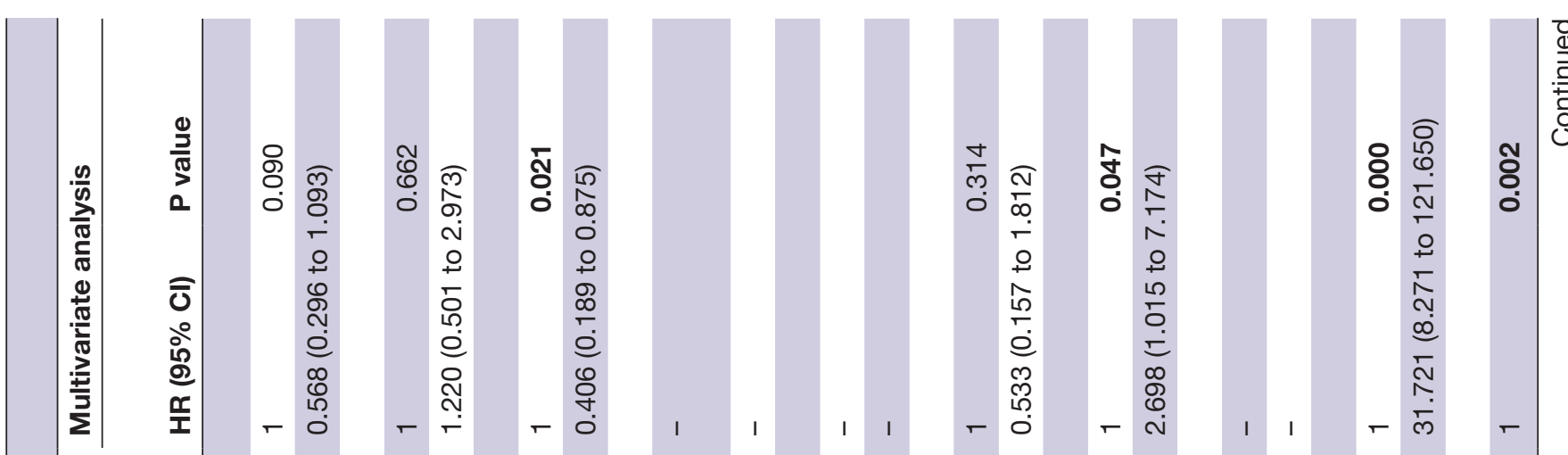

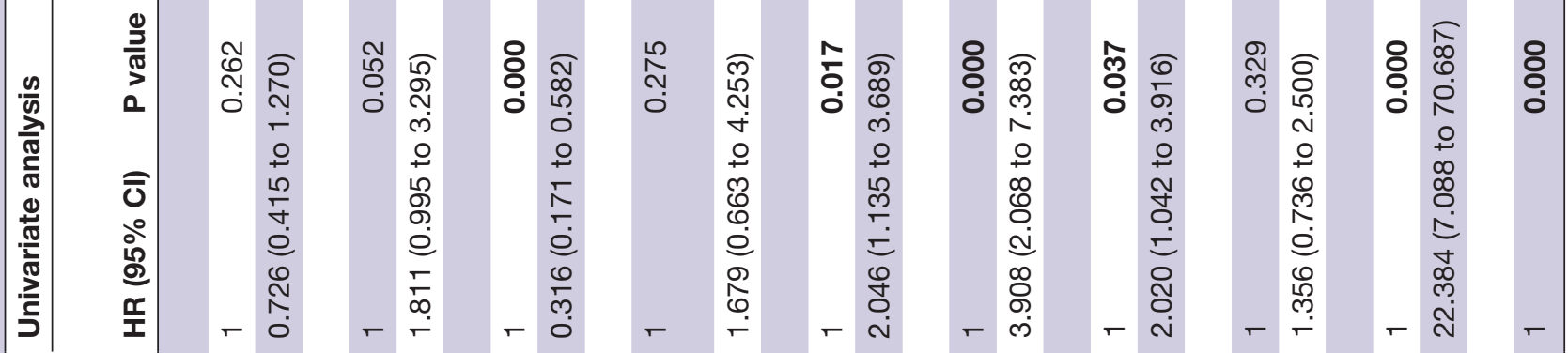

$\frac{\pi}{\sqrt[\pi]{\frac{\pi}{2}}}$

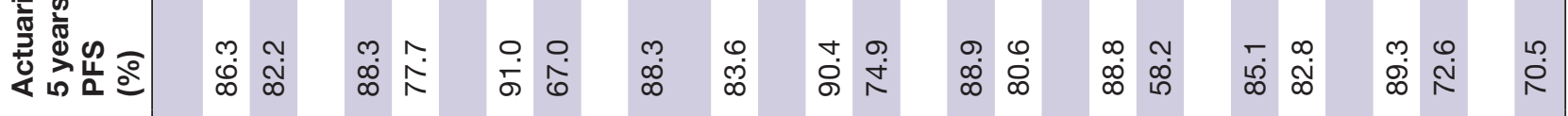

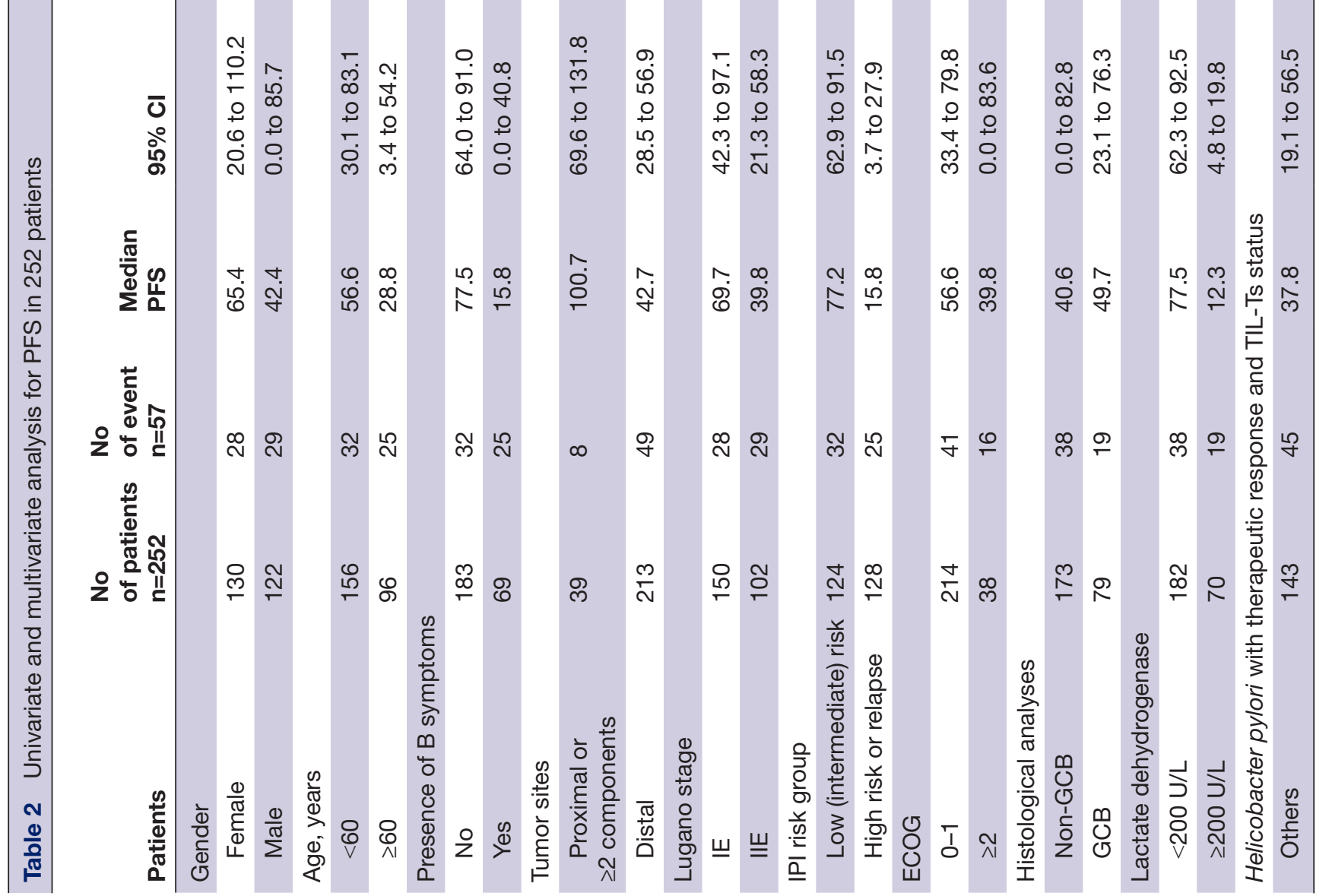




\section{Statistical analyses}

Pearson's $\chi^{2}$ test and Fisher's exact test were applied to determine the correlation between $H$. pylori status and clinicopathological characteristics. The primary endpoints were progression-free survival (PFS) and OS. PFS denotes the length of time from initial diagnosis to disease progression, relapse, or death due to random causes. OS refers to the length of time between the initial diagnosis and death or final records during the follow-up of survivors. The Kaplan-Meier (K-M) method was used to draw the survival curves, and the log-rank test was employed to estimate the significance of differences between the K-M curves. The effective variables in univariate analysis were included in the subsequent multivariate Cox hazards regression analysis for estimating the underlying independent risk factors for PFS and OS. The accurate 5-year PFS and OS were described in the statistical analysis of the 10-year follow-up. The statistical analyses were conducted using SPSS V.25.0 (IBM) and GraphPad Prism V.7 (GraphPad), and the results were presented as means \pm SEM or percentages. Student's t-test was used for comparisons of test subjects from two samples with different conditions. One-way analysis of variance and Tukey's multiple comparisons test were used for multiple comparisons. For all tests, a $\mathrm{p}<0.05$ (two pairs) was considered to indicate significance $(* p<0.05, * * p<0.01$, and $* * * \mathrm{p}<0.001$; not significant (NS) $\mathrm{p}>0.05$ ).

\section{RESULTS}

\section{Patient characteristics}

The baseline clinicopathological parameters of the 252 patients with de novo gDLBCL are listed in table 1 . They were segregated into $H$. pylori positive $(128,50.8 \%)$ and negative (124, 49.2\%) groups. Major gastric ulcer lesions $(68.0 \%$ vs $46.8 \%, p=0.001)$, massive lodging in the proximal stomach (cardia, stomach fundus) $(24.2 \%$ vs $6.5 \%, \mathrm{p}<0.001)$, younger age $(<60 \%, 69.5 \%$ vs $54.0 \%$, $\mathrm{p}=0.011)$, less B symptoms $(89.8 \%$ vs $54.8 \%, \mathrm{p}<0.001)$, higher proportion of normal lactate dehydrogenase (LDH) level $(82 \% .0$ vs $62.1 \%, \mathrm{p}<0.001)$, more GCB origin $(39.1 \%$ vs $23.4 \%, \mathrm{p}=0.016)$, and minor IPI score $(0-1,66.4 \%$ vs $31.5 \%, \mathrm{p}<0.001)$ were correlated with the better prognosis of the H. pylori-positive group. Specially, patients with $H$. pylori infection tended to remain in the IE stage while patients in the $H$. pylori-negative group had a higher proportion of tumors at IIE stage (IE, $72.7 \%$ vs $54.0 \%, \mathrm{p}<0.001)$. There were no significant differences of ECOG, gender, and chemotherapy regimens between the two groups $(p>0.05)$. Intriguingly, more favorable $p C R$ (39.1\% vs $19.4 \%, \mathrm{p}=0.001)$ and higher TIL-Ts $(\geq 14 \%$, $72.7 \%$ vs $46.0 \%, \mathrm{p}=0.012$ ) were noted in the $H$. pyloripositive group.

Over a median follow-up of 66.6 months (range $0.7-119.2$ ), the 5-year OS was $78.0 \%$ for all patients. During the entire study, the median PFS and OS were 49.7 (95\% CI 31.5 to 67.9 ) and 56.2 months (95\% CI 26.6 to 85.8 ), respectively. Significantly favorable 5-year PFS 


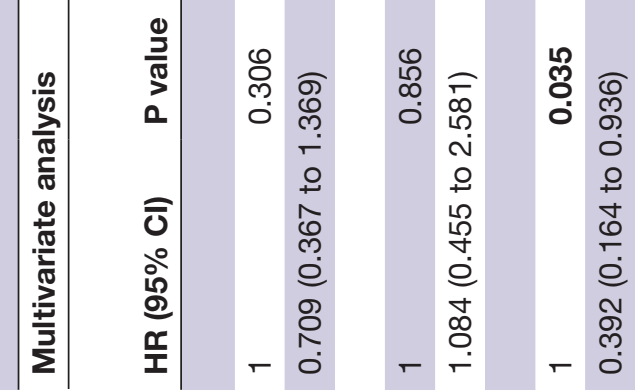
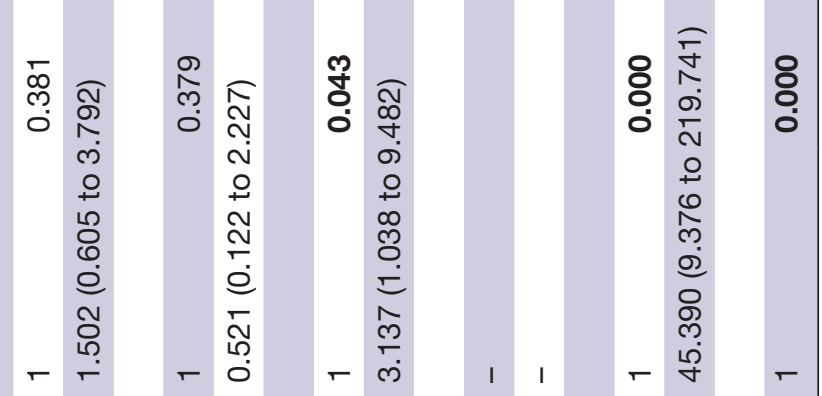

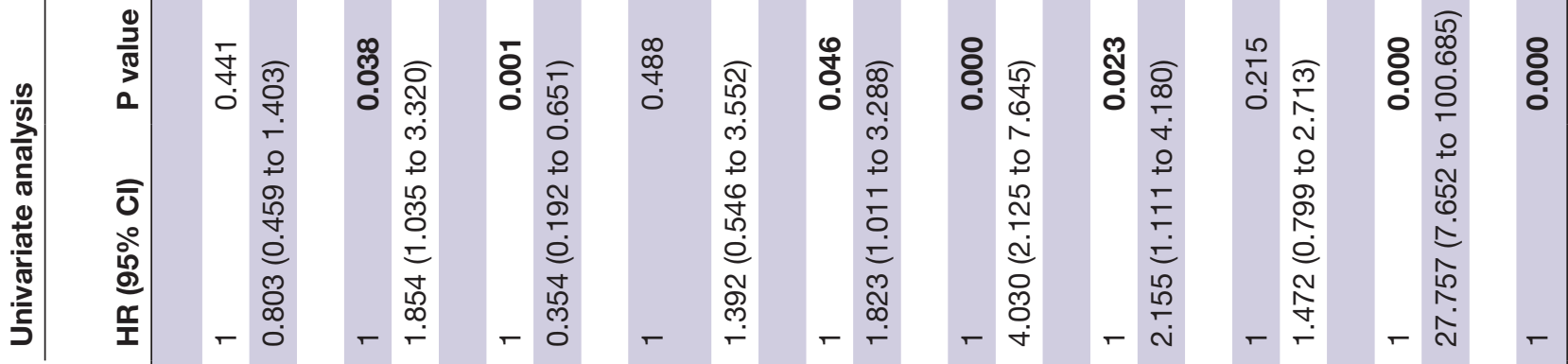

$\overline{\bar{T}}$

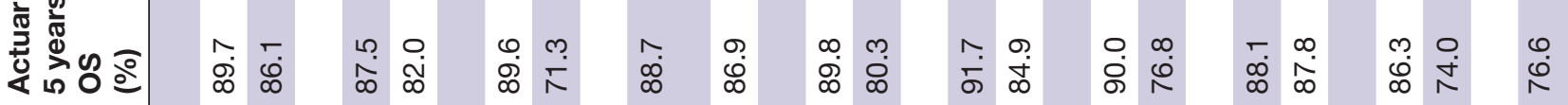

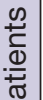

กิ

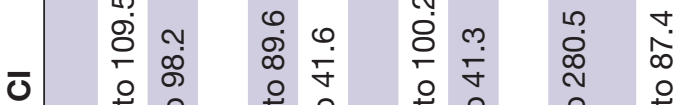

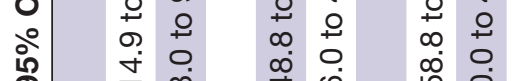

$\begin{array}{ccc}1 & \infty & \\ 0 & \stackrel{0}{0} & \infty \\ 0 & 0 & 1 \\ 0\end{array}$



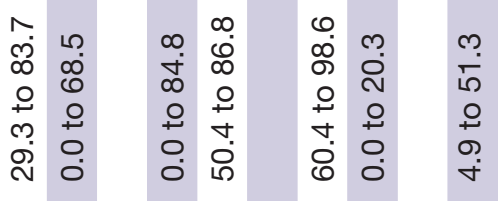

क्ञ

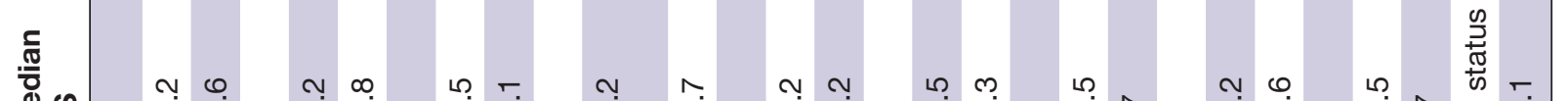

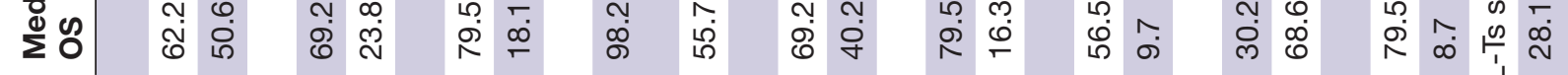

$\frac{\overline{\frac{0}{2}}}{\frac{\overline{0}}{0}}$

$\ddot{2}$

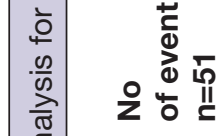
(1)

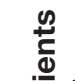

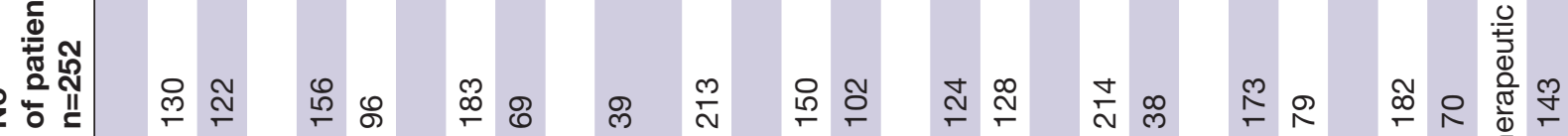
$\mathbf{z}$
$\mathbf{4}$

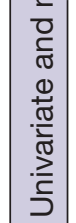

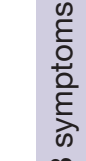

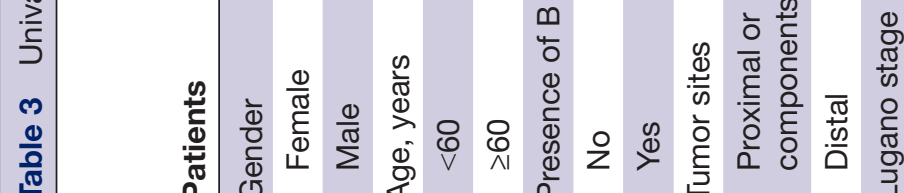




and OS were present in the H. pylori-infected patients with pCR and high-TIL-Ts (termed as 'cohort 1') than in the others (named as 'cohort 2') (5-year PFS, $88.1 \%$ vs $70.5 \%$, $\mathrm{p}<0.001 ; 5$ year OS, $89.2 \%$ vs $76.6 \%, \mathrm{p}<0.001)$, as shown in tables 2 and 3.

H. pylori-positive patients with $\mathrm{pCR}$ and benign TIME had a favorable prognosis of de novo gDLBCL

On follow-up, the median PFS was 120.2 months (95\% CI 93.2 to 147.2 ) for cohort 1 , while it was 37.8 months $(95 \%$ CI 19.1 to 56.5) for cohort $2(\mathrm{p}<0.001)$. The median OS for cohort 1 and cohort 2 was 138.9 months (95\% CI 111.8 to 166.0 ) and 28.1 months (95\% CI 4.9 to 51.3) $(\mathrm{p}<0.001)$, respectively. Univariate analysis demonstrated that the absence of $B$ symptoms $(p<0.001$ for PFS, $p=0.001$ for OS), earlier Lugano stage ( $\mathrm{p}=0.017$ for PFS, $\mathrm{p}=0.046$ for OS), good ECOG state ( $\mathrm{p}=0.037$ for PFS, $\mathrm{p}=0.023$ for OS), low IPI scores ( $p<0.001$ for PFS and OS), normal LDH level ( $\mathrm{p}<0.001$ for PFS and OS), and H. pylori infection with $\mathrm{pCR}$ and high-TIL-Ts $(\mathrm{p}<0.001$ for PFS and OS) were remarkably linked to favorable PFS and OS of de novo gDLBCL. Younger age $(\mathrm{p}=0.038)$ contributed to a lower risk for OS. Multivariate analysis revealed that aberrant LDH levels $(\mathrm{HR}=31.721, \mathrm{p}<0.001$ for PFS; $\mathrm{HR}=45.390, \mathrm{p}<0.001$ for $\mathrm{OS}$ ), weak ECOG performance $(\mathrm{HR}=2.698, \mathrm{p}=0.047$ for $\mathrm{PFS}$; HR=3.137, $\mathrm{p}=0.043$ for $\mathrm{OS})$, and emergent $B$ symptoms (HR=0.406, $\mathrm{p}=0.021$ for PFS; $\mathrm{HR}=0.392, \mathrm{p}=0.035$ for $\mathrm{OS}$ ) were independent predictors of shorter PFS and OS. Importantly, H. pylori-positivity, pCR and high-TIL-Ts state were marked as independent positive prognostic factors for $\mathrm{PFS}(\mathrm{HR}=6.255 ; \mathrm{p}=0.002)$ and $\mathrm{OS}(\mathrm{HR}=26.305 ; \mathrm{p}<0.001) \quad($ tables 2 and 3$)$.

\section{Benign TIME and high response to HPE improved the survival of patients with $\boldsymbol{H}$. pylori-positive de novo gDLBCL}

K-M survival curves indicated that $H$. pylori-positivity with pCR and high-TIL-Ts $(p<0.001)$, young age $(p=0.022)$, absence of $\mathrm{B}$ symptoms $(\mathrm{p}<0.001)$, early Lugano stage $(\mathrm{p}<0.001)$, good ECOG performance status $(\mathrm{p}<0.001)$, regular LDH level $(p<0.001)$, low IPI risk $(p=0.024)$, and proximal location in the stomach $(\mathrm{p}=0.032)$ were significantly correlated with better OS of patients with de novo gDLBCL (figure 1A-H).

\section{Decrease of immunosuppressive components in de novo} gDLBCL of $\boldsymbol{H}$. pylori positive patients with pCR and high TIL-Ts Among 30 subjects, $26 \mathrm{H}$. pylori-positive tissues were confirmed by CagA staining (figure 2A). A total of 19 cases were further defined as the cluster 1 subset according to medical records. They were marked by increased inflammatory cytokines such as TNF- $\alpha$ and IFN- $\gamma$ and decreased immunosuppressive factors such as PD-1, PD-L1, and IL-10 in contrast to the cluster 2 subset (figure 2A,C), and the difference was statistically significant (figure 2B,D). Based on high-quality control of expressed proteins (online supplemental figure 1A-E), a total of 2193 multiple functional proteins covering diverse biological processes were identified by searching 
A

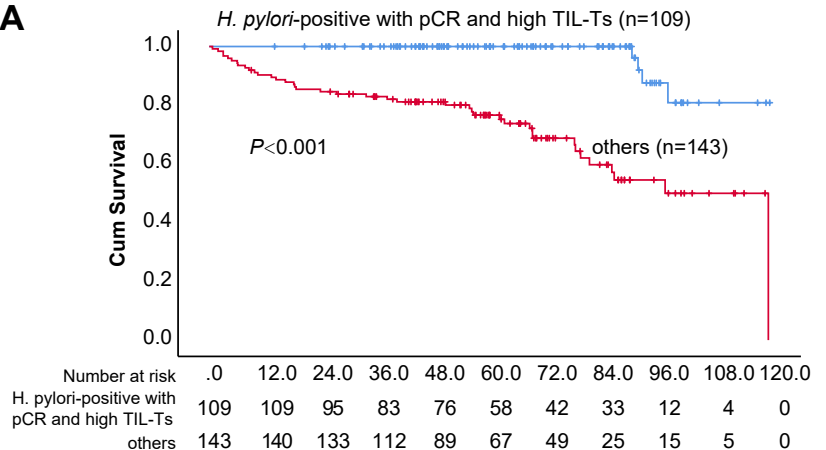

C

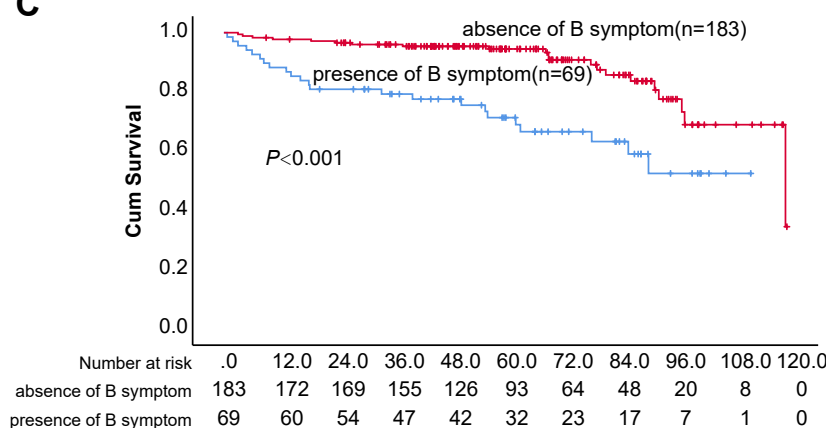

E

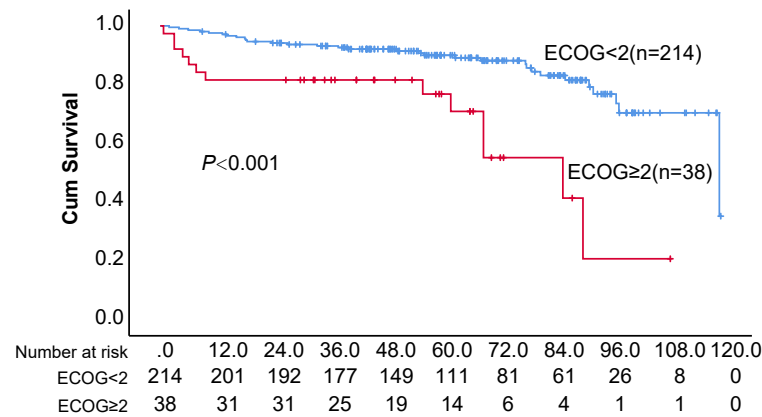

G

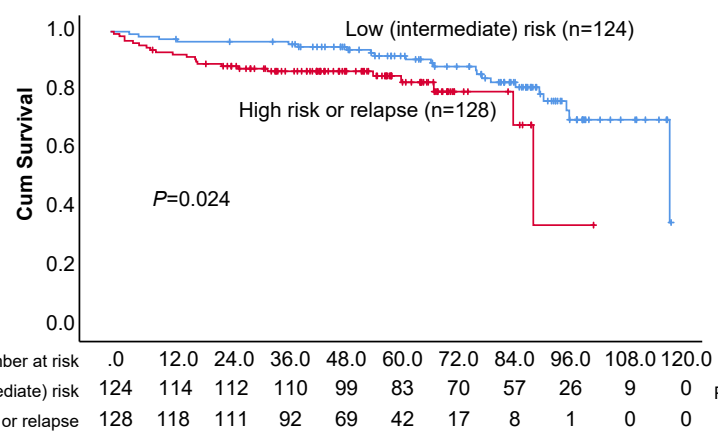

B

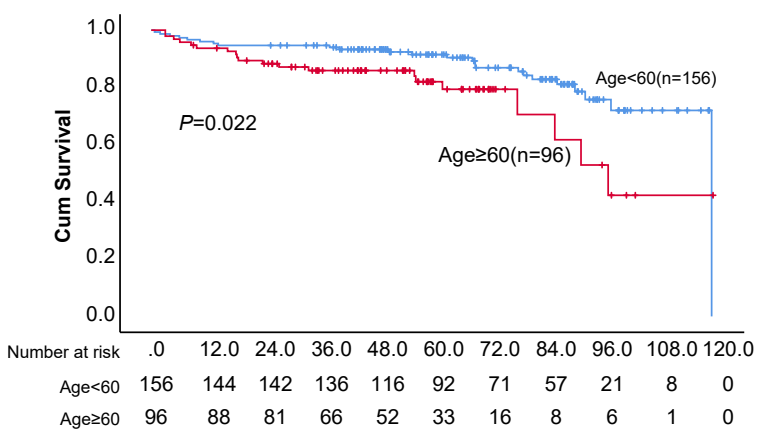

D

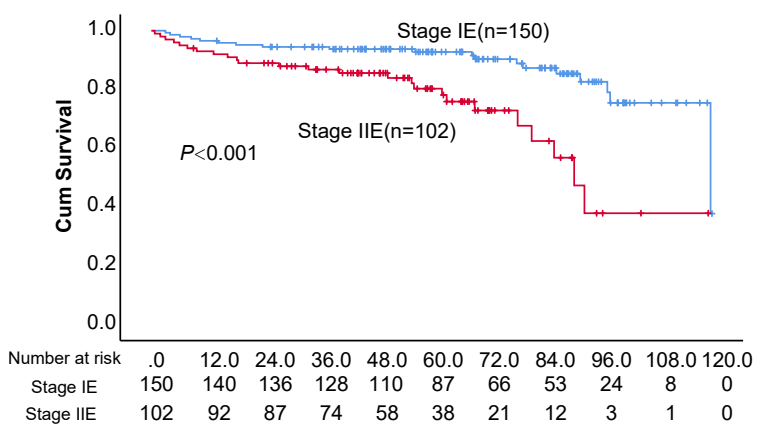

F

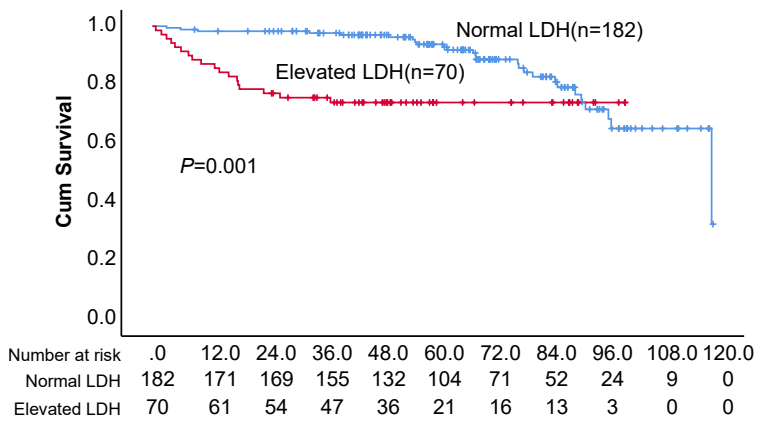

H

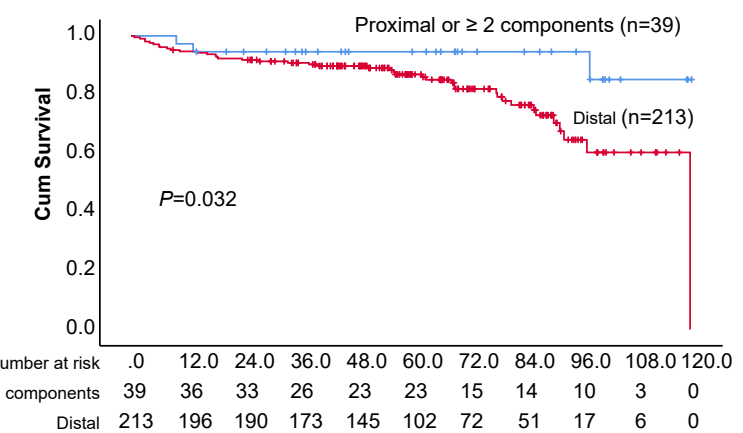

Figure 1 Effects of Helicobacter pylori with pCR and TIL-Ts status, presence of B symptoms, age, ECOG performance, IPI risk group, LDH, Lugano stage and tumor sites on the overall survival of patients with de novo gastric diffuse large B-cell lymphoma (gDLBCL) according to Kaplan-Meier analysis. (A) H. pylori-positive with $\mathrm{pCR}$ and high TIL-Ts $(p<0.001)$, (B) age $<60(\mathrm{p}=0.022)$, (C) absence of $B$ symptoms ( $p<0.001)$, (D) Lugano stage IE ( $p<0.001)$, (E) ECOG $<2$ ( $p<0.001)$, ( $F)$ normal LDH ( $p=0.001)$, (G) low and intermediate risk $(p=0.024)$, (H) proximal or $\geq 2$ components $(p=0.032)$ indicated better os in de novo gDLBCL. Distal, antrum, angle or lower body; ECOG, Eastern Cooperative Oncology Group; IPI, International Prognostic Index; LDH, lactate dehydrogenase; pCR, complete pathological remission; Proximal: middle body, upper body, fundus or cardia; TIL-Ts, tumorinfiltrating T-lymphocytes.

against common databases (online supplemental figure 2A-F). Principal component analysis revealed significant differences of protein abundance between groups, with favorable coefficients of variance (online supplemental figure $1 F, G)$, which were grouped in six expression clusters (figure 3A). Compared with the cluster 2 subset, 
A
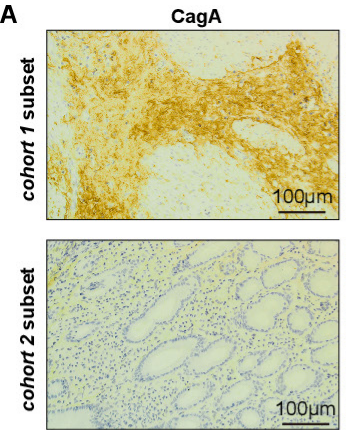

B

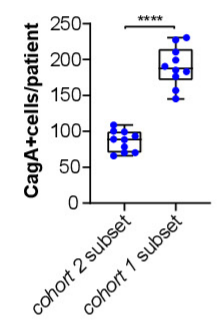

C
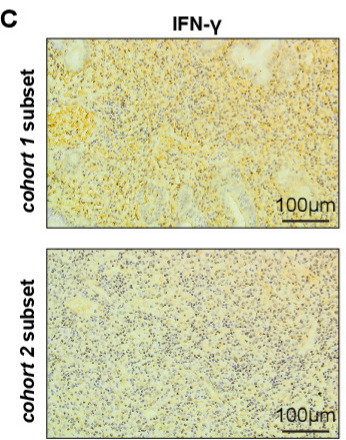

D

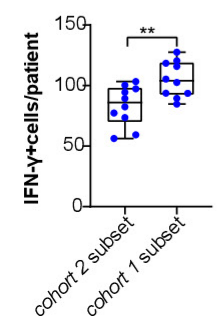

TRAF6
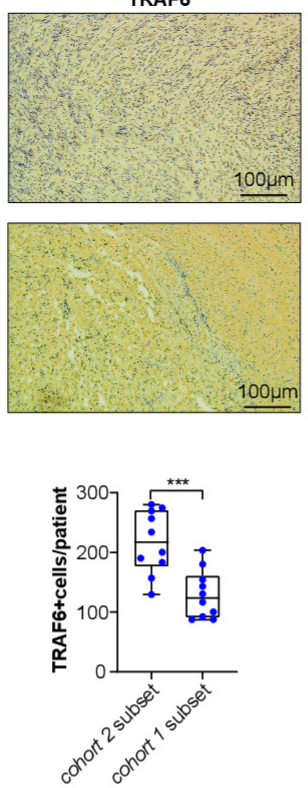

TNF- $\alpha$
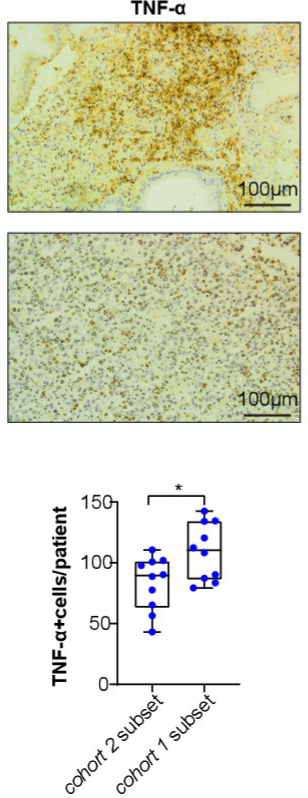

P65
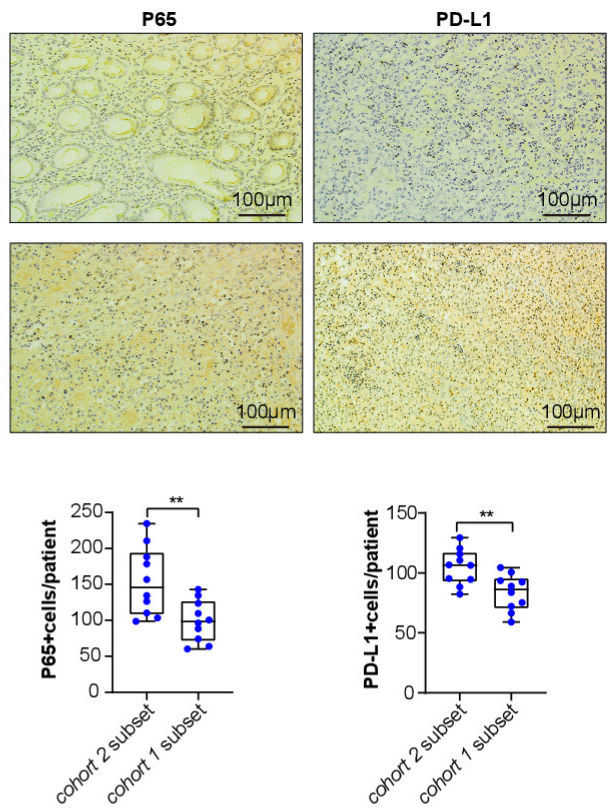

IL-10
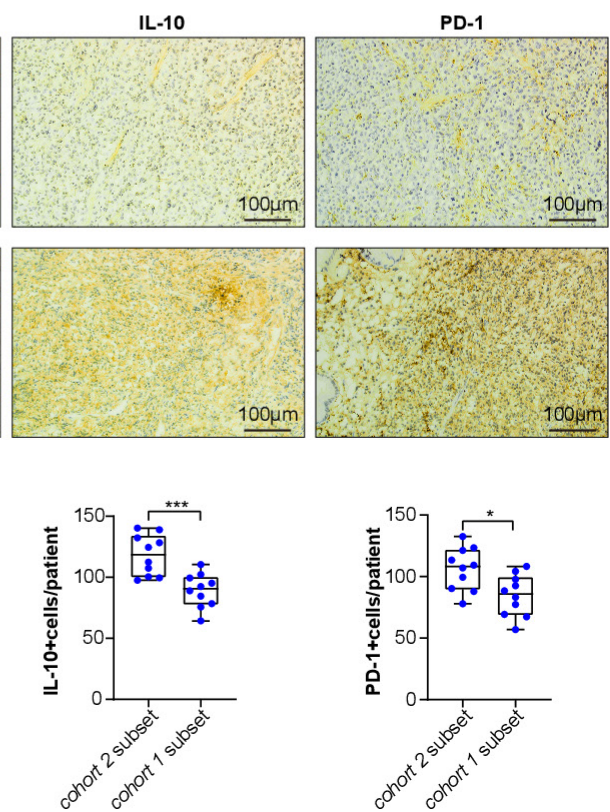

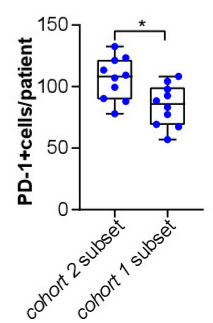

Figure 2 Higher expression of proinflammatory cytokines combined with lower expression of immunosuppressive factors, NF-KB-related proteins and PD-L1 in de novo gDLBCL of Helicobacter pylori-positve patients with pCR and high TIL-Ts. (A, C) Representative immunohistochemistry (IHC) detection of cagA, p65, TRAF6, TNF- $\alpha$, IFN- $\gamma$, PD-L1, PD-1 and IL-10 of cohort 1 subset and cohort 2 subset. Magnification $\times 20$; scale bar: $100 \mu \mathrm{m}$. (B, D) $H$-score of each protein staining degree. Data in graphs are presented as means \pm SEM Statistical analyses by Student's t-test. ${ }^{*} \mathrm{P}<0.05,{ }^{*{ }^{*}} \mathrm{p}<0.01 ;{ }^{* * \star} \mathrm{p}<0.001 ;{ }^{* \star \star *} \mathrm{p}<0.0001$. Cohort 1, H. pylori-positive with PCR and high TIL-Ts; cohort 2, others. gDLBCL, gastric diffuse large B-cell lymphoma; IFN- $\gamma$, interferon $\gamma$, IL-10, interleukin; pCR, complete pathological remission; SEM, SE of the mean; TIL-Ts, tumor-infiltrating Tlymphocytes; TNF- $\alpha$, tumor necrosis factor- $\alpha$.

the cluster 1 subset had reduced immunosuppressive markers such as PD-1, PD-L1, CD206, CD163, IL-10, and TGF- $\beta$ as well as related chemokines such as CCL17, CCL22, CCL5, and CXL5. Conversely, proinflammatory cytokines such as TNF- $\alpha$, IFN- $\gamma$, and IL-12 were comparatively augmented (figure 3B,C). Multidimensional analysis including function, pathways, and structural domains supported the different functional components (figure 4). Specifically, proteins related to the immune response were upregulated $(\mathrm{p}=0.0029)$ (figure $4 \mathrm{~A})$, while the NF-kB $(P<0.001)$ and PI3K-AKT-mTOR $(\mathrm{p}=0.012)$ growth-factor signaling pathways (figure 4B) were downregulated in the cohort 1 subset compared with the cohort 2 subset. Furthermore, intriguing proteins within TIME exhibited characteristic localization and interactions with intergroups differences (online supplemental figure 3 ). These data strongly imply that $H$. pylorielicited inflammatory responses affect the expression of 
A
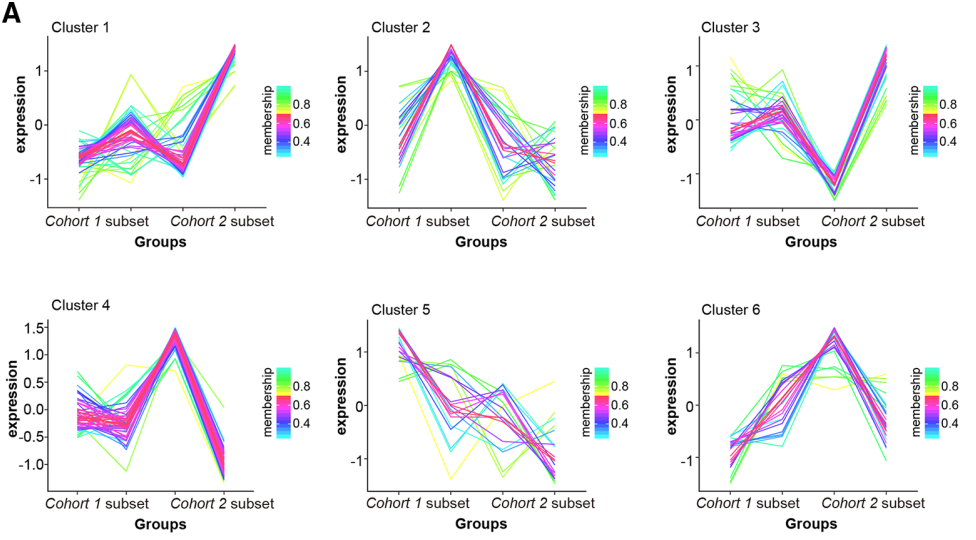

B



C

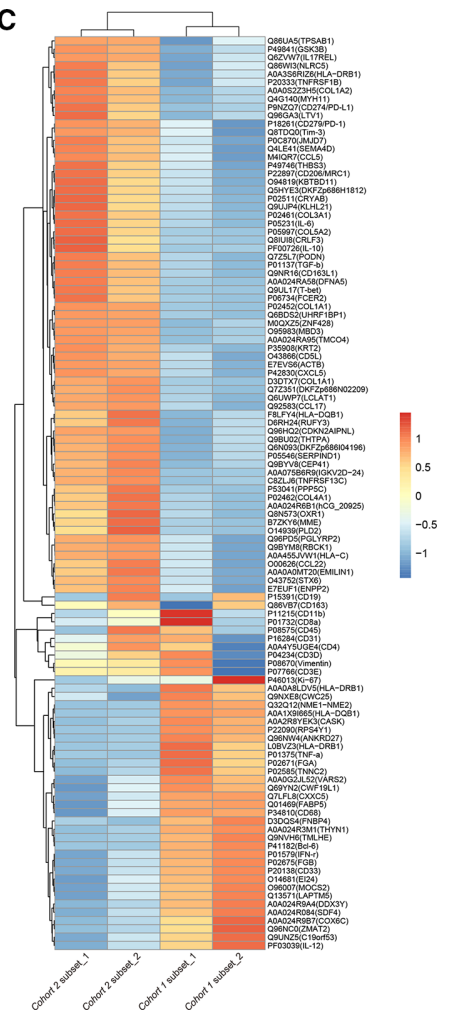

Figure 3 Decreased expression of immunosuppressive proteins in the cohort 1 subset compared with the cohort 2 subset. (A) Immune cells were classified as six clusters in all samples. (B) The volcano plot illustrating the differentially abundant proteins in cohort 1 subset compared with the cohort 2 subset. The $x$ axis specifies the fold-changes (FC) and the $y$ axis specifies the negative logarithm to the base 10 of the t-test $p$ values. Vertical and horizontal lines reflect the filtering criteria ( $F c \geq 1.2$ and $\mathrm{p} \leq 0.05$ for upregulated proteins, $\mathrm{Fc} \leq 0.83$ and $\mathrm{p} \leq 0.05$ for downregulated proteins). Red and green dots represent probe sets for protein expressed at significantly higher (32) and lower (67) levels in the cohort 1 subset compared with the cohort 2 subset, respectively. (C) Heatmap showing the specific upregulated and downregulated immuno-related proteins between the cohort 1 and cohort 2 subsets. Red and blue strips represent significantly higher or lower protein abundance with Z scores ranging from -1 to 1 . The $x$ axis llists the samples and the $y$ axis lists gene identification section (IDS) with corresponding protein names.

immunosuppressive components and reshape the TIME in de novo gDLBCL.

\section{Immune crosstalk in de novo gDLBCL of $H$. pylori-positive tumors with $\mathrm{PCR}$ and high TIL-Ts affects the prognosis}

Based on the prognostic value of TIL-Ts, a holistic and detailed scenario of TIME was developed. The cohort 1 subset had large amounts of aggregated $\mathrm{CD} 45^{+}$immune cells with an equal distribution of myeloid and lymphocytic lineages. Adjacent $\mathrm{CD}^{+}$and $\mathrm{CD}^{+} \mathrm{T}$ cells with similar proportions were located close to the tumor center. Large amounts of effective $\mathrm{CD} 8^{+} \mathrm{T}$ cells partially displayed co-expression of Tim-3 and PD-1, and bound to PD-L1 niches. FOXP3 ${ }^{+}$Tregs, Bcl- $6^{+}$follicular T helper cells, and non-regulatory $\mathrm{CD} 4^{+} \mathrm{CD} 25^{+}$Th cells were in an activated state. CD3-CD56 $6^{+}$natural killer cells were scattered throughout the tissue sections. CD $11 \mathrm{c}^{+} \mathrm{CD} 16^{\text {low }}$ macrophages were in a comparatively immunosuppressed state. Non-tumor CD19+ ${ }^{+}$Bcl- 6 Ki $67^{\text {high }}$ B cells implied low malignancy. The striking contrasts in the cohort 2 subset were characterized by myeloid-dominated clusters with sporadic lymphocytes. Less scattered $\mathrm{T}$ cells maintained the $\mathrm{CD} 8^{+} \mathrm{PD}-1^{-}$phenotype indicative of a static status, restricted by PD-1/PD-L1 modulation due to upregulated tumor PD-L1, and were far away from a few individual $\mathrm{CD}^{+}$cells. CD19 $\mathrm{Bcl}^{+} 6^{+} \mathrm{Ki} 67^{\text {high }}$ tumor B cells suggested high malignancy (figure 5 and online supplemental figure 4).

We quantified the above characteristics and found differences between the two groups (figure 6A,B). After the comparison of density and proximity in each related cluster, the cohort 1 subset had higher proportions of functional immune cells and a closer intracellular spatial distance than the cohort 2 subset, such as S9 for CTL and $\mathrm{S} 10$ for $\mathrm{Th}$, which is associated with better antitumor immunity (figure 6C-F). Taken together, these data demonstrate that $H$. pylori-triggered inflammatory reactions perturbed the TIME in favor of an improved prognosis in de novo gDLBCL.

\section{DISCUSSION}

Despite of pronounced pCR after HPE, ${ }^{17} 18$ there is a wide spectrum of prognosis among $H$. pylori-positive de novo gDLBCL patients. ${ }^{19}$ A study initially proposed that $H$. pylori infection affects the masking of de novo gDLBCL involved in the immune cross-reactions between $H$. pylori and the 
A

GO enrichment Enriched GO Terms (AL)
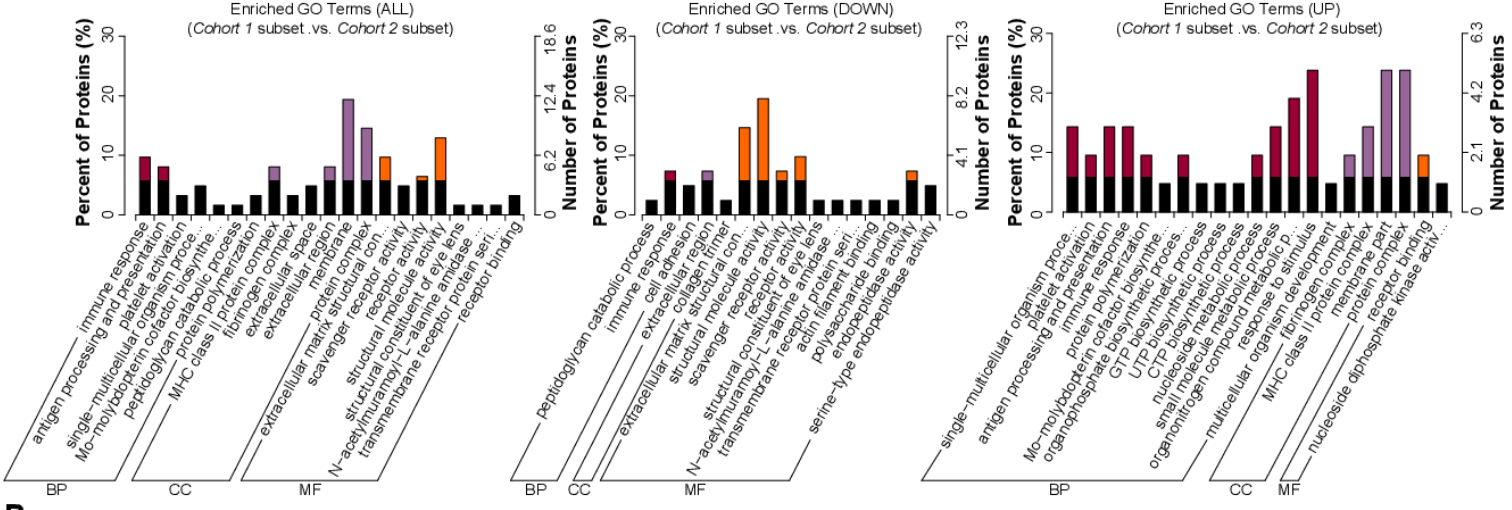

B


Cohort

hort 1 subset .vs. Cohort 2 subset (DOWN)

AGE-RAGE signaling pathway in dabetic complicafions ECM-receptor interaction

Relaxin signaling pathway Signaling pathways regulating pluripotency of stem cells NF-kB signaling pathway Focal adhesion

Glycerophosphdipid metabdism Platelet acfivation

Complement and coagulation cascades Intestinal immune network for IgA production Hepatocellular carcinom Graft-versus-host disease Human papillomavirus infection PI3K-Akt signaling pathway

Influenza $A$ HTLV-I infection 0.
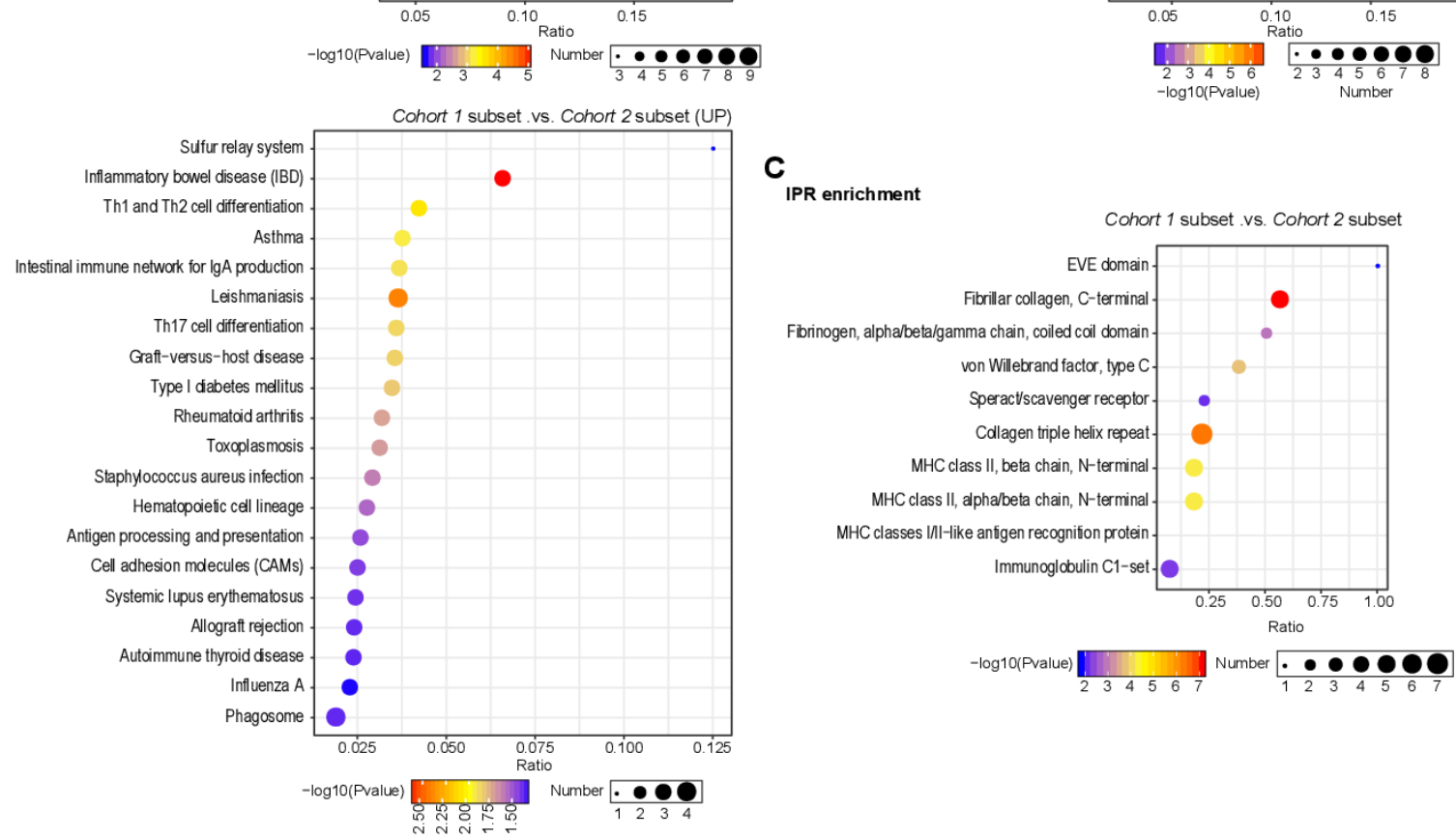

C IPR enrichment

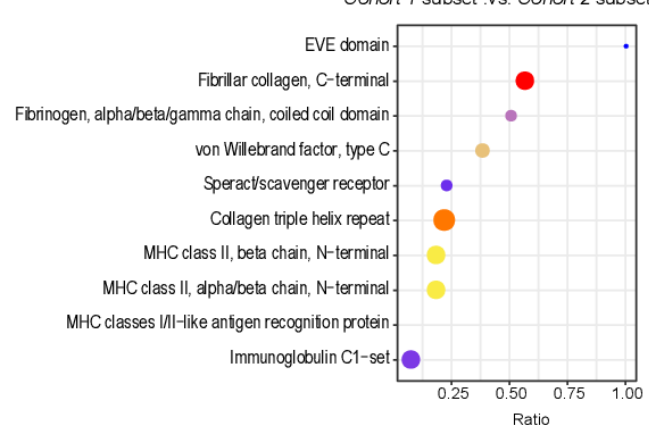

-log10(Pvalue)

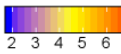

$\int_{7}^{N u m b e r}$ - 0000

Figure 4 GO, KEGG and IPR enrichment analyses of the differentially abundant proteins showing an enhanced immune response and downregulated NF-kB and PI3K-AKT-mTOR growth-factor signaling pathways in the cohort 1 subset compared with the cohort 2 subset. (A) GO enrichment of all, downregulated and upregulated proteins categorized into BP, CC and MF suggested a higher enrichment of immune response-related proteins ( $p=0.0029)$. (B) KEGG enrichment of all, downregulated and upregulated signaling pathways suggested the downregulation of NF-kB $(p<0.001)$ and PI3K-AKT-mTOR growthfactor signaling pathways $(p=0.012)$ in the cohort 1 subset compared with the cohort 2 subset. (C) IPR enrichment of all, downregulated and upregulated IPR categories. BP, biological process; CC, cellular component; GO, Gene Ontology; IPR, InterPro (domain); KEGG, Kyoto Encyclopedia of Genes and Genomes; MF, molecular function. MHC; major histocompatibility complex. EVE; novel pseudouridine synthase and archaeosine transglycosylase (PUA) domain-like domin. 
A
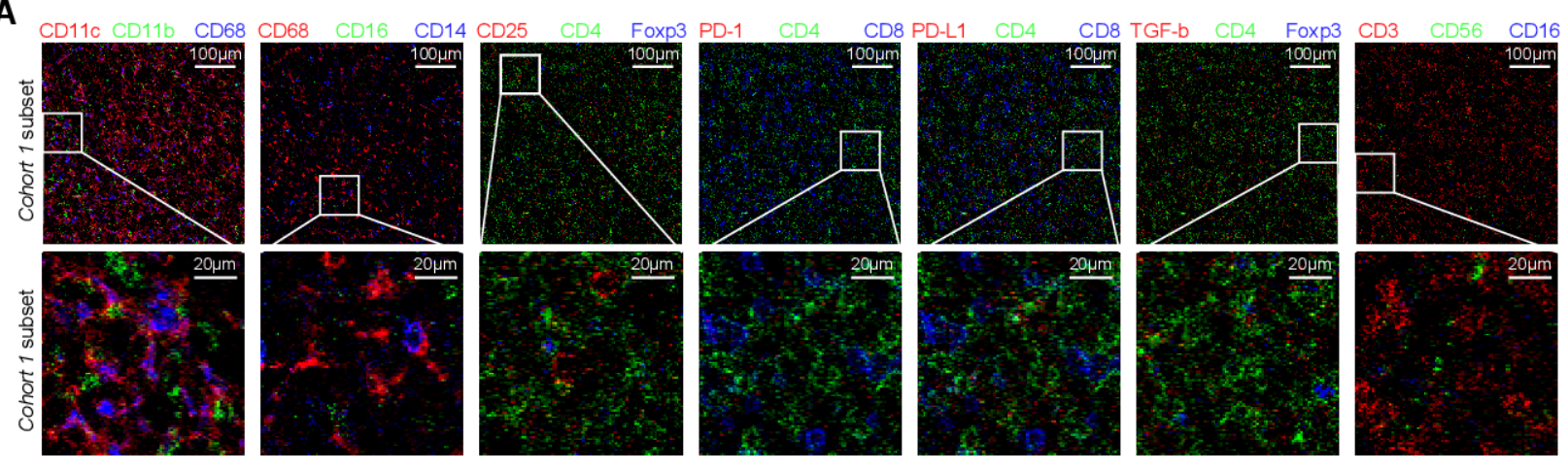

B
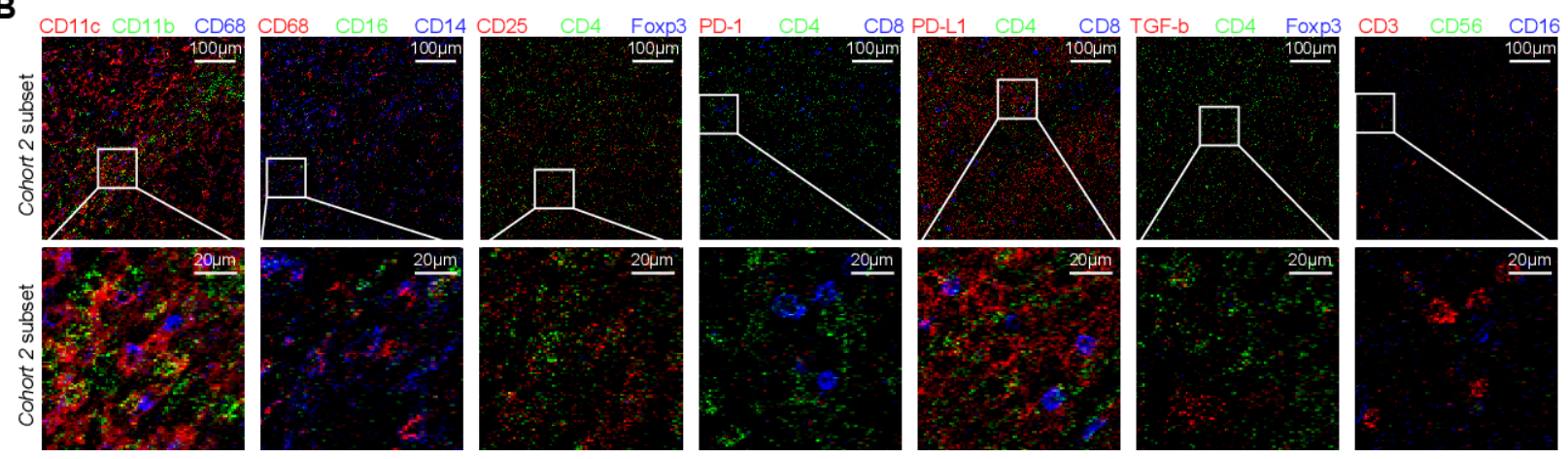

Figure 5 The different spatial distributions of TIME represented by typical immune cell infiltrates between the cohort 1 subset

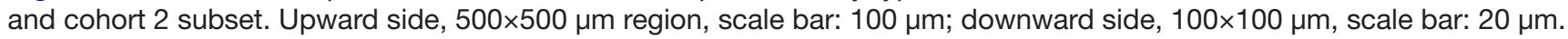
Cohort 1, Helicobacter pylori-positve with pCR and high TIL-Ts; cohort 2, others; TIME, tumor immune microenvironment. pCR, complete pathological remission; TIL-Ts, tumor-infiltrating T-lymphocytes.

B tumor cells. ${ }^{20}$ We found a statistically significant higher proportion of high TIL-Ts counts in $H$. pylori-positive patients than in $H$. pylori-negative ones, preliminarily indicating a benign TIME. The H. pylori-positive patients with pCR and high-TIL-Ts had significantly prolonged survival, emphasizing the impact of the TIME associated with $H$. pylori infection. Previous evidence indicates that PD-1hi T cells promote malignant progression in MALT lymphoma. ${ }^{21}$ We further dissected the precise changes of immune function induced by the $H$. pylori-elicited inflammatory response and TIME by retaining tissues features. Meaningfully, the reduced immunosuppressive molecules and augmented inflammatory components in the cluster 1 subset possibly hint at partial retrieval of antitumor immunity, indicating a relatively benign TIME in the cluster 1.

We proposed that the $H$. pylori-elicited inflammatory response endowed autoimmune Th cells with a longlasting activation status, forming a benign TIME. ${ }^{22}$ When the malignant B-cell-imposed suppressive TIME comes in contact with an active $H$. pylori-induced antitumor inflammatory environment, PD-1/PD-L1-restricted suppressive $\mathrm{T}$ cells or other negative regulators would be impeded, contributing to an ameliorative "cancer-immune set point' and a favorable prognosis. ${ }^{23}$ Conversely, in de novo gDLBCL with an initial refractory or inferior prognosis, ${ }^{24}$ the pace may be such that the formation of tumor-derived TIME lagged behind $H$. pylori-triggered autoimmunity. The tumor effectively performed immune escape, outstripping the diminishing inflammatory activities. In addition, a clinical situation has emerged in which the tumor recurred in spite of a seemingly durable remission at initial treatment. ${ }^{2025}$ One of the possible interpretations is an additional break of cancer-immune homeostasis, ascribed to accelerated time-dependent tumor-imposed immunosuppression, outcompeting the diminishing $H$. pylori-elicited inflammatory immunity over time. Another novel viewpoint focuses on CagA-impacted activation and polarization of T cells. Indeed, CagA elicits Th1-mediated inflammatory immunity and minor Tregs in the earlier stages of gastric carcinogenesis, while the advanced stages are dominated by Th2-mediated inhibitory immunity. ${ }^{26}$ Accordingly, our data showed that the CagA-marked cluster 1 subset displayed expansive $\mathrm{CD} 4^{+} \mathrm{CD} 25^{+} \mathrm{Th} 1$ cell populations. This observation deepened our understanding of the time-dependent changes of the TIME, which directly governs cancer progression.

We intuitively illustrated the prognostic determinants based on immune-crosstalk insights. ${ }^{14} 27$ Antitumor immunity was subdivided into the immune-desert phenotype with deficiency of immune activation, the immuneexclusion phenotype with specific immune barriers, and the inflamed phenotype with massive immunocyte infiltration, ${ }^{28}$ reflecting the divergent clinical outcomes. ${ }^{29} 30$ Based on an analysis of the spatial distributions of the TIME at initial diagnosis without clinical intervention, we reasoned that cohort 1 represented inflamed tumors with more infiltration by functional immune cells. We 


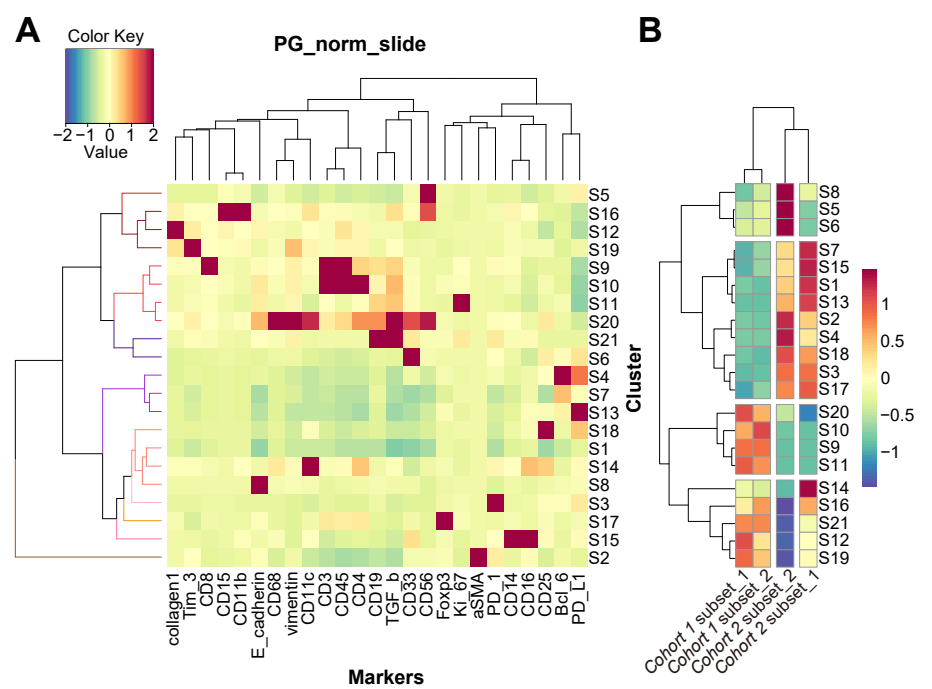

C

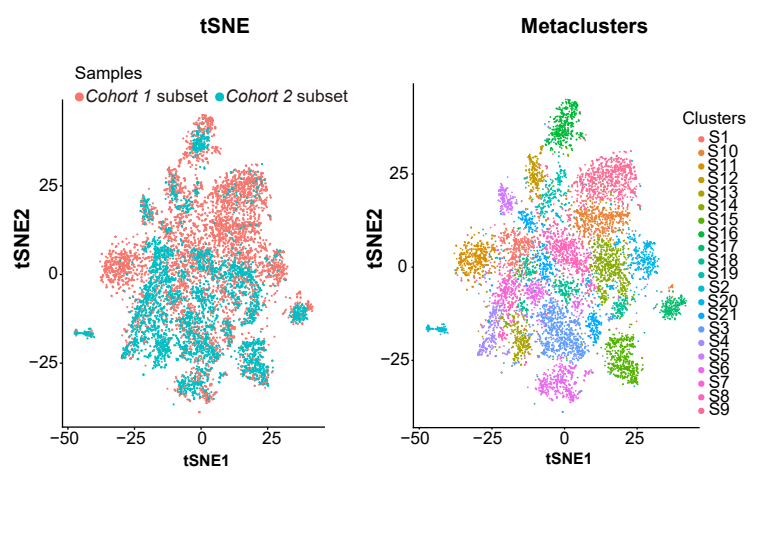

D

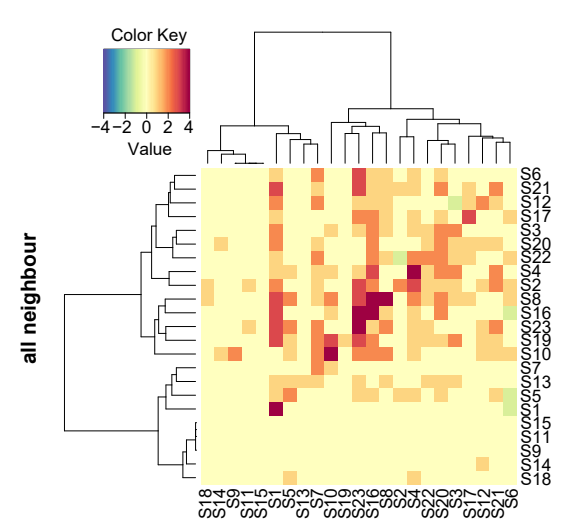

$\mathbf{F}$

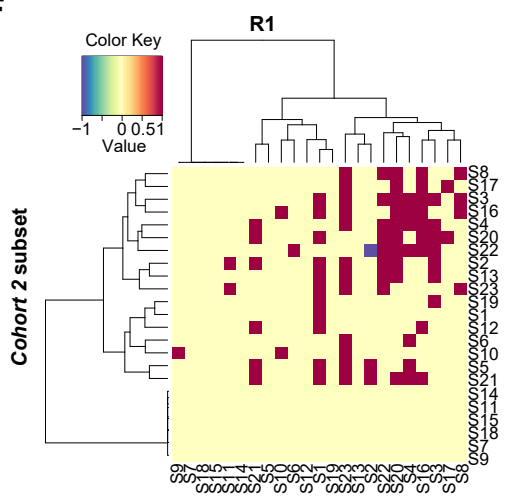

E


Figure 6 (A) Tumor cells and infiltrating immune cells in the TIME from all regions were clustered according to protein expression markers (total 21). The expression values of each protein are scaled from -2 to 2 . (B) The different expression values of 21 resulting clusters ( $y$ axis) between the cohort 1 subset and cohort 2 subset $(x$ axis) is shown, scaled from -1 to 1. (C) tSNE embedding for tumor cells and infiltrating immune cells in the time. Cells are colored by either the sample (left) or cluster (right). Heatmaps showing the spatial proximity $z$ scores between pairs of clusters in regions of all neighbors $(D)$, in selected regions of $\mathrm{R} 1$ and R2 of the cohort 1 subset (E) as well as those of the cohort 2 subset (F). TIME, tumor immune microenvironment.

accordingly defined cohort 2 as representing immunedesert tumors due to only a few sporadic T cells. More $\mathrm{CD}^{+} \mathrm{T}$ cells got closer to the tumor center and were adjacent to other immune cells in the cohort 1 subset. This was associated with better survival, similar to a precious report for DLBCL. ${ }^{31}$ This spatial distinction of the TIME was in agreement with the idea of a disturbance due to
H. pylori infection and its pivotal role in the ameliorative prognosis.

Given the above evidence that the underlying determinants of survival in de novo gDLBCL are related to the crosstalk within paradoxical microenvironments derived from tumor-mediated and $H$. pylori-induced states, we felt that this provides novel key points for treatment 
strategies based on an evaluation of $H$. pylori and TIME status. H. pylori-elicited spatiotemporal diversification of the TIME poses a challenge in ascertaining the optimal therapeutic opportunity. Faced with relapse in a subset of $H$. pylori-positive patients following conventional treatment, ${ }^{32}$ it pointed to the caveat that the effect of TIME is non-negligible and could be used to stratify patients for personalized treatment. At present, the Food and Drug Administration (FDA) has approved two chimeric antigen receptor-T medicines targeting relapsed and refractory $\mathrm{B}$ cell lymphoma. ${ }^{33}$ It is further highlighted that not only $H$. pylori status but also the diverse TIME should be taken into consideration for better clinical outcomes in de novo gDLBCL. Given the limited patient samples and other unknown adaptive intrinsic differences derived from H. pylori-evoked biological behaviors, ${ }^{34}$ the influence of $H$. pylori on the TIME and its correlation with the prognosis should be further verified in a larger cohort. By all accounts, this study is the first to illustrate the mechanism of $H$. pylori-related prognosis in de novo gDLBCL, providing valuable insights for novel clinical treatment directions.

\section{CONCLUSIONS}

In summary, $H$. pylori-elicited inflammatory responses may improve the prognosis via crosstalk with the TIME in de novo gDLBCL. $H$. pylori infection with PCR and high-TIL-Ts can hence be termed as an independent prognostic factor for de novo gDLBCL and help identify patients that will benefit from precision treatment.

\section{Author affiliations \\ ${ }^{1}$ Department of Medical Oncology, Harbin Medical University Cancer Hospital, Harbin, Heilongjiang, People's Republic of China \\ ${ }^{2}$ Department of Hematology, First Affiliated Hospital of Harbin Medical University, Harbin, Heilongjiang, China \\ ${ }^{3}$ Institute of Cancer Prevention and Treatment, Heilongjiang Academy of Medical Sciences, Harbin Medical University, Harbin, Heilongjiang, People's Republic of China \\ ${ }^{4}$ Department of Pathology, Harbin Medical University Cancer Hospital, Harbin, Heilongjiang, People's Republic of China}

Acknowledgements Harbin Xingyun Medical Inspection Institute and Beijing Jinkorui Medical Laboratory provided technical assistance. The clinical data collection was supported by the Harbin Medical University Cancer Hospital and the First-affiliated Hospital of Harbin Medical University.

Collaborators Not avaliable.

Contributors YD and WS contribute equally in this study.

Funding This study was financed by a major project of the National Natural Science Foundation of China (No. 81730074) and N10 project in the Cancer Hospital of Harbin Medical University.

Competing interests No, there are no competing interests.

Patient consent for publication Consent obtained directly from patient(s)

Ethics approval All study procedures were approved by the institutional review boards of the Harbin Medical University Cancer Hospital and First-affiliated Hospital of Harbin Medical University. This study was performed in accordance with the Declaration of Helsinki.

Provenance and peer review Not commissioned; externally peer reviewed.

Data availability statement Data are available on reasonable request.
Supplemental material This content has been supplied by the author(s). It has not been vetted by BMJ Publishing Group Limited (BMJ) and may not have been peer-reviewed. Any opinions or recommendations discussed are solely those of the author(s) and are not endorsed by BMJ. BMJ disclaims all liability and responsibility arising from any reliance placed on the content. Where the content includes any translated material, BMJ does not warrant the accuracy and reliability of the translations (including but not limited to local regulations, clinical guidelines, terminology, drug names and drug dosages), and is not responsible for any error and/or omissions arising from translation and adaptation or otherwise.

Open access This is an open access article distributed in accordance with the Creative Commons Attribution Non Commercial (CC BY-NC 4.0) license, which permits others to distribute, remix, adapt, build upon this work non-commercially, and license their derivative works on different terms, provided the original work is properly cited, appropriate credit is given, any changes made indicated, and the use is non-commercial. See http://creativecommons.org/licenses/by-nc/4.0/.

\section{ORCID iD}

Qingyuan Zhang http://orcid.org/0000-0001-8116-5293

\section{REFERENCES}

1 Cuccurullo R, Govi S, Ferreri AJM. De-escalating therapy in gastric aggressive lymphoma. World J Gastroenterol 2014;20:8993-7.

2 Kotlov N, Bagaev A, Revuelta MV, et al. Clinical and biological subtypes of B-cell lymphoma revealed by microenvironmental signatures. Cancer Discov 2021;11:1468-89.

3 Li Q, Li W, Wang L, et al. Consolidation radiotherapy in stage IE- IIE, Non-Bulky primary gastric diffuse large B-cell lymphoma with postchemotherapy complete remission. PLoS One 2015;10:e0133469.

4 Lin L-C, Hsu T-H, Huang K-W, et al. Nonbismuth concomitant quadruple therapy for Helicobacter pylori eradication in Chinese regions: a meta-analysis of randomized controlled trials. World $J$ Gastroenterol 2016;22:5445-53.

5 Ferreri AJM, Govi S, Ponzoni M. The role of Helicobacter pylori eradication in the treatment of diffuse large B-cell and marginal zone lymphomas of the stomach. Curr Opin Oncol 2013;25:470-9.

6 Paydas S. Helicobacter pylori eradication in gastric diffuse large B cell lymphoma. World J Gastroenterol 2015;21:3773-6.

7 Juárez-Salcedo LM, Sokol L, Chavez JC, et al. Primary gastric lymphoma, epidemiology, clinical diagnosis, and treatment. Cancer Control 2018;25:107327481877825.

8 Kang HJ, Lee HH, Jung S-E, et al. Pattern of failure and optimal treatment strategy for primary gastric diffuse large B-cell lymphoma treated with R-CHOP chemotherapy. PLoS One 2020;15:e0238807.

9 Cheng Y, Xiao Y, Zhou R, et al. Prognostic significance of Helicobacter pylori-infection in gastric diffuse large B-cell lymphoma. BMC Cancer 2019;19:842.

10 Kuo S-H, Yeh K-H, Wu M-S, et al. Helicobacter pylori eradication therapy is effective in the treatment of early-stage $\mathrm{H}$ pylori-positive gastric diffuse large B-cell lymphomas. Blood 2012;119:4838-44.

11 Cioroianu Al, Stinga PI, Sticlaru L, et al. Tumor microenvironment in diffuse large B-cell lymphoma: role and prognosis. Anal Cell Pathol 2019;2019:1-9.

12 Ishikawa E, Nakamura M, Shimada K, et al. Prognostic impact of $\mathrm{PD}-\mathrm{L} 1$ expression in primary gastric and intestinal diffuse large B-cell lymphoma. J Gastroenterol 2020;55:39-50.

13 Vincent-Fabert C, Roland L, Zimber-Strobl U, et al. Pre-Clinical blocking of PD-L1 molecule, which expression is down regulated by NF-KB, JAK1/JAK2 and Btk inhibitors, induces regression of activated B-cell lymphoma. Cell Commun Signal 2019;17:89.

14 Dixon BREA, Hossain R, Patel RV, et al. Th17 cells in Helicobacter pylori infection: a dichotomy of help and harm. Infect Immun 2019;87:e00363-19.

15 Kuo S-H, Chen L-T, Lin C-W, et al. Expressions of the CagA protein and CagA-signaling molecules predict Helicobacter pylori dependence of early-stage gastric DLBCL. Blood 2017;129:188-98.

16 Chen Z, Deng X, Ye Y, et al. Novel risk stratification of de novo diffuse large $B$ cell lymphoma based on tumour-infiltrating $T$ lymphocytes evaluated by flow cytometry. Ann Hematol 2019;98:391-9.

17 Violeta Filip P, Cuciureanu D, Sorina Diaconu L, et al. Malt lymphoma: epidemiology, clinical diagnosis and treatment. $J$ Med Life 2018;11:187-93.

18 Ferreri AJM, Govi S, Ponzoni M. The role of Helicobacter pylori eradication in the treatment of diffuse large B-cell and marginal zone lymphomas of the stomach. Curr Opin Oncol 2013;25:470-9.

19 Saito M, Mori A, Ogasawara R, et al. Progression of primary gastric diffuse large B-cell lymphoma after Helicobacter pylori eradication. Case Rep Gastroenterol 2020;14:534-9. 
20 Kuo S-H, Yeh K-H, Chen L-T, et al. Helicobacter pylori-related diffuse large B-cell lymphoma of the stomach: a distinct entity with lower aggressiveness and higher chemosensitivity. Blood Cancer $J$ 2014:4:e220.

21 Wickenden K, Nawaz N, Mamand S, et al. PD ${ }^{\text {hi }}$ cells associate with clusters of proliferating B-cells in marginal zone lymphoma. Diagn Pathol 2018;13:74.

22 Bergman MP, D'Elios MM. Cytotoxic T cells in H. pylori-related gastric autoimmunity and gastric lymphoma. J Biomed Biotechnol 2010;2010:1-10.

23 Falchi L. Immune dysfunction in non-Hodgkin lymphoma: avenues for new immunotherapy-based strategies. Curr Hematol Malig Rep 2017;12:484-94.

24 Ikoma N, Badgwell BD, Mansfield PF. Multimodality treatment of gastric lymphoma. Surg Clin North Am 2017:97:405-20.

25 Zelenetz AD, Gordon LI, Wierda WG, et al. Non-Hodgkin's lymphomas, version 4.2014. J Natl Compr Canc Netw 2014;12:1282-303.

26 Dixon BREA, Hossain R, Patel RV, et al. Th17 cells in Helicobacter pylori infection: a dichotomy of help and harm. Infect Immun 2019;87:e00363-19.

27 Capitani N, Codolo G, Vallese F, et al. The lipoprotein HP1454 of Helicobacter pylori regulates T-cell response by shaping T-cell receptor signalling. Cell Microbiol 2019;21:e13006.
28 Chen DS, Mellman I. Elements of cancer immunity and the cancerimmune set point. Nature 2017;541:321-30.

29 Papin A, Le Gouill S, Chiron D. Rationale for targeting tumor cells in their microenvironment for mantle cell lymphoma treatment. Leuk Lymphoma 2018;59:1064-72.

30 Lavin Y, Kobayashi S, Leader A, et al. Innate immune landscape in early lung adenocarcinoma by paired single-cell analyses. Cell 2017;169:750-65.

31 Shi Y, Deng L, Song Y, et al. CD3+/CD8+ T-cell density and tumoral PD-L1 predict survival irrespective of rituximab treatment in Chinese diffuse large B-cell lymphoma patients. Int $\mathrm{J}$ Hematol 2018;108:254-66.

32 Yang $\mathrm{H}$, Wu M, Shen $\mathrm{Y}$, et al. Treatment strategies and prognostic factors of primary gastric diffuse large B cell lymphoma: a retrospective multicenter study of 272 cases from the China lymphoma patient registry. Int J Med Sci 2019;16:1023-31.

33 Ok CY, Young KH. Checkpoint inhibitors in hematological malignancies. J Hematol Oncol 2017;10:103.

34 Ishikura $\mathrm{N}$, Usui $\mathrm{Y}$, Ito $\mathrm{H}$, et al. Helicobacter pylori (HP) infection alone, but not HP-induced atrophic gastritis, increases the risk of gastric lymphoma: a case-control study in Japan. Ann Hematol 2019;98:1981-7. 